\title{
EVIDENCE FOR AN FU ORIONIS-LIKE OUTBURST FROM A CLASSICAL T TAURI STAR
}

\author{
Adam A. Miller ${ }^{1}$, Lynne A. Hillenbrand ${ }^{2}$, Kevin R. Covey ${ }^{3,7,8}$, Dovi Poznanski ${ }^{1,4,9}$, Jeffrey M. Silverman ${ }^{1}$, \\ Io K. W. Kleiser ${ }^{1}$, Bárbara Rojas-Ayala ${ }^{3}$, Philip S. Muirhead ${ }^{3}$, S. Bradley Cenko ${ }^{1}$, Joshua S. Bloom ${ }^{1}$, \\ Mansi M. Kasliwal ${ }^{2}$, Alexei V. Filippenko ${ }^{1}$, Nicholas M. LaW ${ }^{5}$, Eran O. Ofek ${ }^{2,9}$, Richard G. Dekany ${ }^{6}$, \\ Gustavo Rahmer $^{6}$, David Hale ${ }^{6}$, Roger Smith ${ }^{6}$, Robert M. Quimby ${ }^{2}$, Peter Nugent ${ }^{4}$, Janet Jacobsen ${ }^{4}$, Jeff Zolkower ${ }^{6}$,

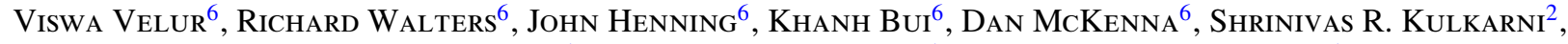 \\ Christopher R. Klein ${ }^{1}$, Michael Kandrashoff ${ }^{1}$, and AleKzandir Morton ${ }^{1}$ \\ ${ }^{1}$ Department of Astronomy, University of California, Berkeley, CA 94720-3411, USA \\ ${ }^{2}$ Astrophysics Department, California Institute of Technology, Pasadena, CA 91125, USA \\ ${ }^{3}$ Department of Astronomy, Cornell University, Ithaca, NY 14853, USA \\ ${ }^{4}$ Computational Cosmology Center, Lawrence Berkeley National Laboratory, 1 Cyclotron Road, Berkeley, CA 94720, USA \\ ${ }^{5}$ Dunlap Institute for Astronomy and Astrophysics, University of Toronto, 50 St. George Street, Toronto, M5S 3H4 Ontario, Canada \\ ${ }^{6}$ Caltech Optical Observatories, California Institute of Technology, Pasadena, CA 91125, USA \\ Received 2010 November 9; accepted 2011 January 24; published 2011 March 8
}

\begin{abstract}
We present pre- and post-outburst observations of the new FU Orionis-like young stellar object PTF 10qpf (also known as $\mathrm{LkH} \alpha 188-\mathrm{G} 4$ and $\mathrm{HBC}$ 722). Prior to this outburst, $\mathrm{LkH} \alpha$ 188-G4 was classified as a classical T Tauri star (CTTS) on the basis of its optical emission-line spectrum superposed on a K8-type photosphere and its photometric variability. The mid-infrared spectral index of $\mathrm{LkH} \alpha$ 188-G4 indicates a Class II-type object. LkH $\alpha$ 188-G4 exhibited a steady rise by $\sim 1$ mag over $\sim 11$ months starting in August 2009, before a subsequent more abrupt rise of $>3$ mag on a timescale of $\sim 2$ months. Observations taken during the eruption exhibit the defining characteristics of FU Orionis variables: (1) an increase in brightness by $\gtrsim 4$ mag, (2) a bright optical/near-infrared reflection nebula appeared, (3) optical spectra are consistent with a $\mathrm{G}$ supergiant and dominated by absorption lines, the only exception being $\mathrm{H} \alpha$ which is characterized by a P Cygni profile, (4) near-infrared spectra resemble those of late $\mathrm{K}-\mathrm{M}$ giants/supergiants with enhanced absorption seen in the molecular bands of $\mathrm{CO}$ and $\mathrm{H}_{2} \mathrm{O}$, and (5) outflow signatures in $\mathrm{H}$ and $\mathrm{He}$ are seen in the form of blueshifted absorption profiles. $\mathrm{LkH} \alpha 188$-G4 is the first member of the FU Orionis-like class with a well-sampled optical to mid-infrared spectral energy distribution in the pre-outburst phase. The association of the PTF 10qpf outburst with the previously identified CTTS LkH $\alpha$ 188-G4 (HBC 722) provides strong evidence that FU Orionis-like eruptions represent periods of enhanced disk accretion and outflow, likely triggered by instabilities in the disk. The early identification of PTF 10qpf as an FU Orionis-like variable will enable detailed photometric and spectroscopic observations during its post-outburst evolution for comparison with other known outbursting objects.
\end{abstract}

Key words: stars: formation - stars: individual (LkHa 188-G4, HBC 722) - stars: pre-main sequence - stars: variables: T Tauri, Herbig Ae/Be - stars: winds, outflows

Online-only material: color figures

\section{INTRODUCTION}

FU Orionis variables are young stellar objects (YSOs) that exhibit large-amplitude optical outbursts $\left(\Delta m_{\text {visual }} \gtrsim 4 \mathrm{mag}\right)$ and remain bright for several decades (Herbig 1977). Models in which the radiation is dominated by a rapid increase in the disk accretion rate have successfully reproduced many of the observational properties of FU Orionis outbursts; see Hartmann \& Kenyon (1996) for a review. An alternative interpretation involving a rapidly rotating low-gravity star is presented by Herbig (1989) and Herbig et al. (2003). In the first scenario, the cause of the instability remains uncertain; possible mechanisms include thermal instabilities (Hartmann \& Kenyon 1996) and interactions with companion stars on eccentric orbits (Bonnell $\&$ Bastien 1992). Accretion instabilities are also believed to give

\footnotetext{
7 Hubble Fellow.

8 Visiting Researcher, Department of Astronomy, Boston University, 725 Commonwealth Ave., Boston, MA 02215, USA.

9 Einstein Fellow.
}

rise to less dramatic YSO flares. ${ }^{10}$ These events (e.g., EX Lupi, V1647 Ori, V1118 Ori, Z CMa; see Herbig 1977, 2007; Aspin et al. 2010; Aspin \& Reipurth 2009; Fedele et al. 2007; Rettig et al. 2005; Lorenzetti et al. 2007; Audard et al. 2010; Szeifert et al. 2010; and references therein) are heterogeneous, and it remains to be determined if they should be referred to as a single class (Herbig 2007). These YSO outbursts are nevertheless distinguished from FU Orionis-like outbursts in that their optical spectra are emission-line-dominated (whereas FU Ori stars typically show only absorption lines during outburst) and the outbursts have shorter lifetimes, $\tau_{\text {outburst }} \lesssim 2 \mathrm{yr}$, than FU Ori stars which remain in outburst for decades (Herbig 1977; Fedele et al. 2007).

YSO outbursts are of great interest given their potential importance to the broader star and planet formation process. Low-mass stars could accrete as much as half their final mass

\footnotetext{
${ }^{10}$ In the literature, these events are frequently referred to as "EXors," named for the outbursting star EX Lupi. This nomenclature is confusing, however, as EX Lupi is not part of the Orion constellation and many of the so-called EXors seem to have distinct properties (see, e.g., Fedele et al. 2007; Herbig 2007; Covey et al. 2011).
} 
during FU Orionis outbursts (Hartmann \& Kenyon 1996), and the strong winds and outflows they are thought to drive likely have a significant impact on the surrounding interstellar medium (Croswell et al. 1987).

One of the major challenges in studying FU Orionis stars is the relatively small sample of known examples. Although $\sim 20$ FU Orionis candidates have been identified by Reipurth \& Aspin (2010) based on a mix of spectroscopic evidence, only a handful $(\sim 6-8)$ of those stars have been observed to rise from their pre-outburst state to their eruptive state. Only one example, V1057 Cyg, has had a pre-outburst spectrum taken; it showed Balmer, Ca II, Fe I, and Fe II lines in emission (Herbig 1977). Herbig noted that these features resembled those of a $\mathrm{T}$ Tauri star, but a lack of detected absorption lines precluded a spectral-type classification beyond a late-type star based on the red continuum.

Recently, a new FU Orionis-like variable, associated with $\mathrm{LkH} \alpha 188-\mathrm{G} 4$, was discovered at $\alpha_{\mathrm{J} 2000}=20^{\mathrm{h}} 58^{\mathrm{m}} 17^{\mathrm{s}} .00$, $\delta_{\mathrm{J} 2000}=+43^{\circ} 53^{\prime} 42^{\prime \prime} .9$ in the direction of NGC 7000/IC 5070, also known as the North America/Pelican Nebula. The source was announced as a likely FU Ori-like outburst on 2010 August $17 \mathrm{UT}^{11}$ by Semkov \& Peneva (2010a) and was later spectroscopically confirmed as an FU Ori candidate by Munari et al. (2010). Further photometric observations showed that the source became bluer over the course of the outburst and that a reflection nebula was emerging around $\mathrm{LkH} \alpha$ 188-G4 (Semkov \& Peneva 2010b). Near-infrared (NIR) photometric observations revealed a $\sim 3$ mag increase in the $J, H$, and $K_{s}$ bands (Leoni et al. 2010). The variable was independently discovered by our collaboration during the course of regular monitoring of the North America Nebula with the Palomar 48 in telescope. These images were automatically reduced, and new variable and transient sources were found using the software developed for the Palomar Transient Factory (PTF; Law et al. 2009; Rau et al. 2009; J. S. Bloom et al. 2011, in preparation). On 2010 August 5, the outburst from $\mathrm{LkH} \alpha$ 188-G4 was identified and given the name PTF 10qpf.

Here, we present pre- and post-eruption observations of $\mathrm{LkH} \alpha$ 188-G4, an FU Orionis-like star, which we argue was a classical T Tauri star (CTTS) prior to eruption. Shortly before the submission of this article, an independent analysis of $\mathrm{LkH} \alpha$ 188-G4 was posted on the arXiv by Semkov et al. (2010), in which they also argue that the 2010 eruption from $\mathrm{LkH} \alpha 188$ G4 is an FU Orionis outburst. In Section 2, we discuss archival observations of $\mathrm{LkH} \alpha 188-\mathrm{G} 4$ prior to the 2010 eruption. Our new 2009 and 2010 observations are presented in Section 3, and Section 4 contains our analysis of those observations. We discuss our conclusions in Section 5. Throughout this paper, we designate the 2010 outburst as PTF 10qpf, while we refer to the pre-outburst star as $\mathrm{LkH} \alpha$ 188-G4.

\section{PRE-OUTBURST OBSERVATIONS OF LkH $\alpha$ 188-G4}

PTF 10qpf is associated with the previously known optical source LkH $\alpha$ 188-G4 (Cohen \& Kuhi 1979), which was later cataloged by Herbig \& Bell (1988) as HBC 722. Cohen \& Kuhi (1979) identified the star as an $m_{V}=18.9$ mag emissionline object with line equivalent widths of $\mathrm{EW}_{\mathrm{H} \alpha}=-100.5 \AA$ and $\mathrm{EW}_{\mathrm{H} \beta}=-25.8 \AA$; [OI] $\lambda 6300$ was also present, and NIR photometry was reported. We are unable to identify the strong emission feature near $4640 \AA$ in the Cohen \& Kuhi (1979) spectrum, and the authors themselves do not measure

\footnotetext{
11 UT dates are used throughout unless otherwise noted.
}

Table 1

IPHAS Observations of $\mathrm{LkH} \alpha$ 188-G4

\begin{tabular}{lccc}
\hline \hline UT Date & $\begin{array}{c}r \text { mag } \\
(\mathrm{AB})\end{array}$ & $\begin{array}{c}i \text { mag } \\
(\mathrm{AB})\end{array}$ & $\begin{array}{c}\mathrm{H} \alpha \text { mag } \\
(\mathrm{AB})\end{array}$ \\
\hline 2003 Oct 15 & $17.300 \pm 0.010$ & $15.677 \pm 0.006$ & $16.023 \pm 0.007$ \\
2003 Oct 18 & $17.348 \pm 0.011$ & $15.744 \pm 0.006$ & $16.063 \pm 0.006$ \\
2003 Nov 2 & $17.283 \pm 0.017$ & $15.608 \pm 0.009$ & $15.975 \pm 0.009$ \\
2003 Nov 14 & $17.074 \pm 0.009$ & $15.570 \pm 0.005$ & $15.724 \pm 0.006$ \\
2005 Nov 19 & $17.075 \pm 0.007$ & $15.671 \pm 0.006$ & $15.768 \pm 0.008$
\end{tabular}

or comment on the feature. It could be related to multiply ionized C or N, or He II (see, e.g., Sargent \& Filippenko 1991), though the absence of a known exact wavelength for the feature precludes a secure identification. The original spectrum is no longer available (M. Cohen 2010, private communication) and we caution that the feature may be an error in the original data. Cohen \& Kuhi (1979) determined a spectral type of K7-M0; they also derived an extinction estimate of $A_{V}=3.4 \pm 1.2 \mathrm{mag}$ and a bolometric luminosity of $\log \left(L / L_{\odot}\right)=0.43$.

The source is part of a linear chain of YSOs that was first designated by Cohen \& Kuhi (1979) as the NGC 7000/ IC $5070 \mathrm{LkH} \alpha 188$ group. Spitzer IRAC and MIPS observations presented by Guieu et al. (2009) and Rebull et al. (2011) later revealed that the optically visible stars are only the surface population of a much richer embedded cluster, known as the "Gulf of Mexico" cluster due to its location relative to the North America Nebula. The Two Micron All Sky Survey (2MASS; Skrutskie et al. 2006) observed the field in the $J, H$, and $K_{s}$ bands on 2000 June 10 . BVI optical photometry obtained with the Kitt Peak National Observatory $0.9 \mathrm{~m}$ telescope was presented by Guieu et al. (2009), while broadband $r i$ and narrowband $\mathrm{H} \alpha$ imaging of $\mathrm{LkH} \alpha$ 188-G4, obtained between 2003 and 2005 , is presented in the catalog of the INT/WFC Photometric $\mathrm{H} \alpha$ Survey of the Northern Galactic Plane (IPHAS; GonzálezSolares et al. 2008). We summarize the IPHAS measurements in Table 1.

Owing to crowding, there are no IRAS measurements of $\mathrm{LkH} \alpha$ 188-G4. Additional IR sources are cataloged by the Midcourse Space Experiment (MSX; Mill et al. 1994) and $A K A R I$ (Murakami et al. 2007). The $M S X$ source has a position $\left(\alpha_{\mathrm{J} 2000}=20^{\mathrm{h}} 58^{\mathrm{m}} 17^{\mathrm{s}} .59, \delta_{\mathrm{J} 2000}=43^{\circ} 53^{\prime} 36^{\prime \prime} .24\right)$ which is $\sim 9^{\prime \prime} .2$ from the optically derived position of $\mathrm{LkH} \alpha$ 188-G4. This position has no clear counterpart, to within $\sim 3^{\prime \prime}$, in the 2 MASS catalog, and thus the $M S X$ source may represent a blend of emission from several stars in the $\mathrm{LkH} \alpha 188$ group. There are two sources in the AKARI MIR catalog (Ishihara et al. 2010) within $10^{\prime \prime}$ of the optical position of $\mathrm{LkH} \alpha 188-\mathrm{G} 4$. Both of these sources are $>4^{\prime \prime} .5$ from the optical position of $\operatorname{LkH} \alpha 188$ G4, however, so we do not include them here in our analysis of pre-outburst emission from $\mathrm{LkH} \alpha 188-\mathrm{G} 4$; the photometry of these sources likely does not correspond to $\mathrm{LkH} \alpha 188-\mathrm{G} 4$ alone. AKARI also catalogs a source in the far-infrared (FIR) at $\alpha_{\mathrm{J} 2000}=20^{\mathrm{h}} 58^{\mathrm{m}} 16^{\mathrm{s}} .809, \delta_{\mathrm{J} 2000}=+43^{\circ} 53^{\prime} 41^{\prime \prime} .65$, which is $\sim 2$. 4 from the optical position of $\mathrm{LkH} \alpha 188-\mathrm{G} 4$. The closest 2MASS counterpart to the AKARI FIR source is LkH $\alpha 188-\mathrm{G} 4$, though we cannot rule out the possibility that the $A K A R I$ flux measurements include a contribution from other members of the LkH $\alpha 188$ group.

The pre-outburst spectral energy distribution (SED) of $\mathrm{LkH} \alpha$ 188-G4 is shown in Figure 1. The pre-outburst SED of $\mathrm{LkH} \alpha$ 188-G4 exhibits a smooth trend with the exception of the two points at $\sim 0.75$ and $0.9 \mu \mathrm{m}$. These points, corresponding to the 


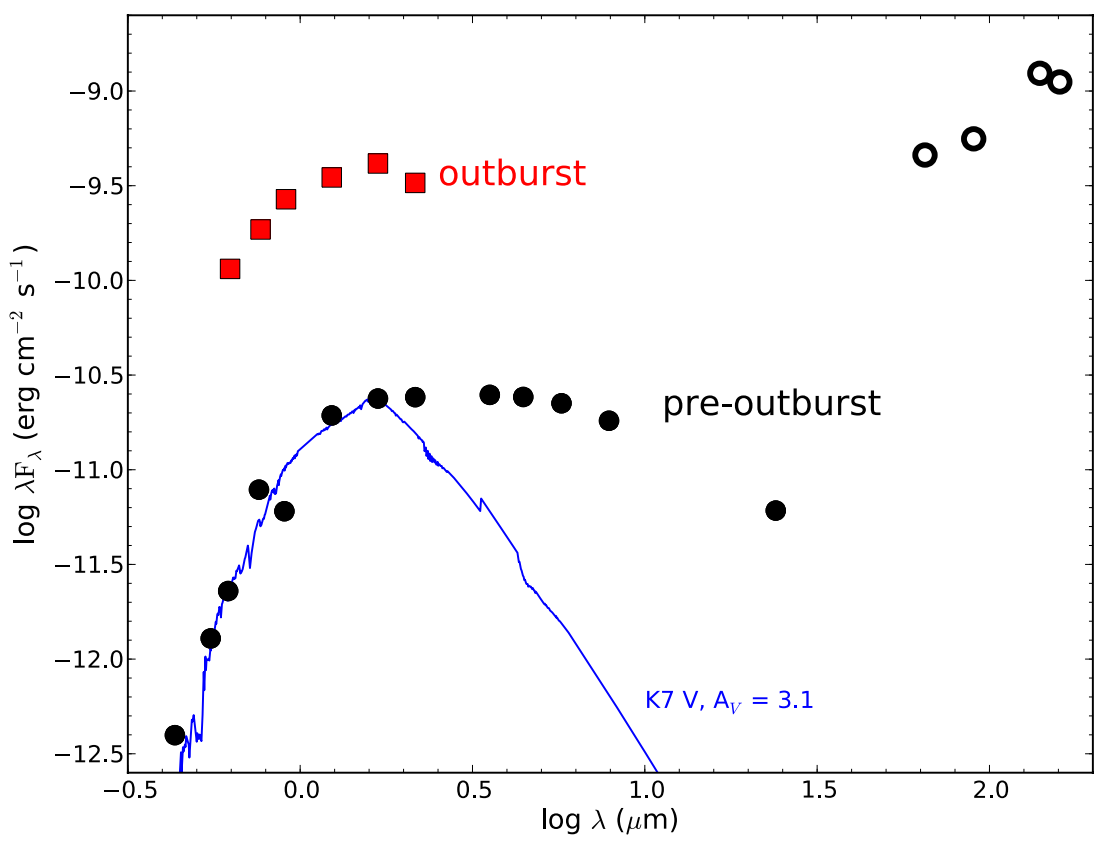

Figure 1. Spectral energy distribution of $\mathrm{LkH} \alpha 188$-G4 both before (black circles) and during (red squares) the 2010 outburst. Pre-outburst NIR photometry is from 2MASS, while non-simultaneous optical and MIR photometry is from Guieu et al. (2009), Rebull et al. (2011), and González-Solares et al. (2008). FIR photometry from AKARI is shown as open circles because these detections may include light from other members of the LkH $\alpha 188$ group (see the text and Figure 3). The solid line shows the model spectrum, from Kurucz (1993), of a K7 V star reddened with $A_{V}=3.1 \mathrm{mag}$, and demonstrates the infrared excess of LkH $\alpha$ 188-G4. The spectral slope in the MIR is consistent with the source being a Class II YSO prior to outburst. NIR outburst photometry was obtained on 2010 October 10 with PAIRITEL, while riz photometry was taken on 2010 October 14 with P60.

(A color version of this figure is available in the online journal.)

IPHAS $i$ band and KPNO $I$ band, respectively, are not coeval and differ by a factor of $\sim 30 \%$ in flux, which is similar to the $\sim 0.3$ mag variability seen in the multi-epoch IPHAS data.

Direct integration of the pre-outburst SED, excluding the $A K A R I$ data, results in a total luminosity of $\sim 0.7 L_{\odot}$, assuming a distance of $600 \mathrm{pc}$ to NGC 7000. Adopting the classification scheme from Lada \& Wilking (1984), we fit for the MIR spectral index, defined as

$$
\alpha=\frac{d \log \lambda F_{\lambda}}{d \log \lambda}
$$

where $\alpha$ is the spectral index and $F_{\lambda}$ is the flux density at wavelength $\lambda$. Following a least-squares fit to the Spitzer/IRAC data from 3.6 to $8 \mu \mathrm{m}$, we measure $\alpha=-0.40$, indicating that $\mathrm{LkH} \alpha$ 188-G4 was a Class II YSO prior to its 2010 outburst. Thus, there was no evidence of either a dense core of infalling material or significant envelope material in the vicinity of the central star. Including the Spitzer/MIPS $24 \mu \mathrm{m}$ detection results in $\alpha=-0.77$, securely establishing this source as a Class II YSO. To demonstrate the MIR excess relative to a stellar photosphere, we also show in Figure 1 the model spectrum of a solar metallicity $\mathrm{K} 7 \mathrm{~V}$ star, reddened with $A_{V}=3.1 \mathrm{mag}$, which is consistent with the inferences from Cohen \& Kuhi (1979). The model spectrum, taken from the Kurucz (1993) update to the Kurucz (1979) models, has been normalized to match the flux of $\mathrm{LkH} \alpha 188-\mathrm{G} 4$ in the $H$ band.

Class II YSOs are most commonly associated with CTTSs (Lada 1987). In addition to being a Class II YSO, we also know from the multi-epoch IPHAS data that LkH $\alpha$ 188-G4 was optically variable between 2003 and 2005, years before the observed PTF outburst, with an amplitude of $\sim 0.2-0.3$ mag. While some CTTSs show variable amplitudes as large as $\sim 2 \mathrm{mag}$, the majority exhibit low-amplitude variability consistent with what we observe in LkH $\alpha$ 188-G4 prior to eruption (Grankin et al.
2007). The emission-line spectrum, Class II MIR spectral index, and optical variability all point to $\mathrm{LkH} \alpha 188-\mathrm{G} 4$ being a fairly undistinguished CTTS prior to its 2010 eruption.

\section{2009 AND 2010 OBSERVATIONS}

\subsection{Optical Photometry and an Independent Discovery}

During the 2009 and 2010 observing seasons, PTF obtained red optical images of the North America/Pelican Nebula starforming region with a typical 5 day cadence. These observations were conducted with the main PTF Survey Camera, the former CFHT12K mosaic camera now extensively re-engineered and mounted on the 48 inch Samuel Oschin Telescope at Palomar Observatory (hereafter P48). The camera is a mosaic of 12 CCDs (one of which is not functional), covering a $7.8 \mathrm{deg}^{2}$ field of view with $1^{\prime \prime}$ sampling; typical conditions at Palomar Observatory produce 2 .'0 full width at half-maximum (FWHM) intensity images (Law et al. 2009). The $R_{\mathrm{PTF}}$ filter, a Mould $R$ band, is similar to the Sloan Digital Sky Survey (SDSS) $r$ band in shape but is shifted redward by $\sim 27 \AA$ and is $\sim 20 \AA$ wider. The typical $5 \sigma$ limiting magnitudes are $m_{R} \approx 20.6(\mathrm{AB})$ in $60 \mathrm{~s}$ exposures.

Transient sources are detected in the PTF-monitoring data by means of automated reduction pipelines, including a nearly realtime image-subtraction pipeline hosted at Lawrence Berkeley National Laboratory (LBNL). Well-detected sources in the difference images are scored (using a human-trained, machinebased classifier) for their likelihood of being truly astrophysical in nature or of spurious origin. Variable sources with larger likelihoods of being nonspurious are passed to an automatic source classifier at UC Berkeley (Oarical), which combines PTF measurements with all other available information (e.g., SIMBAD identifications, 2MASS photometry, etc.) to provide 


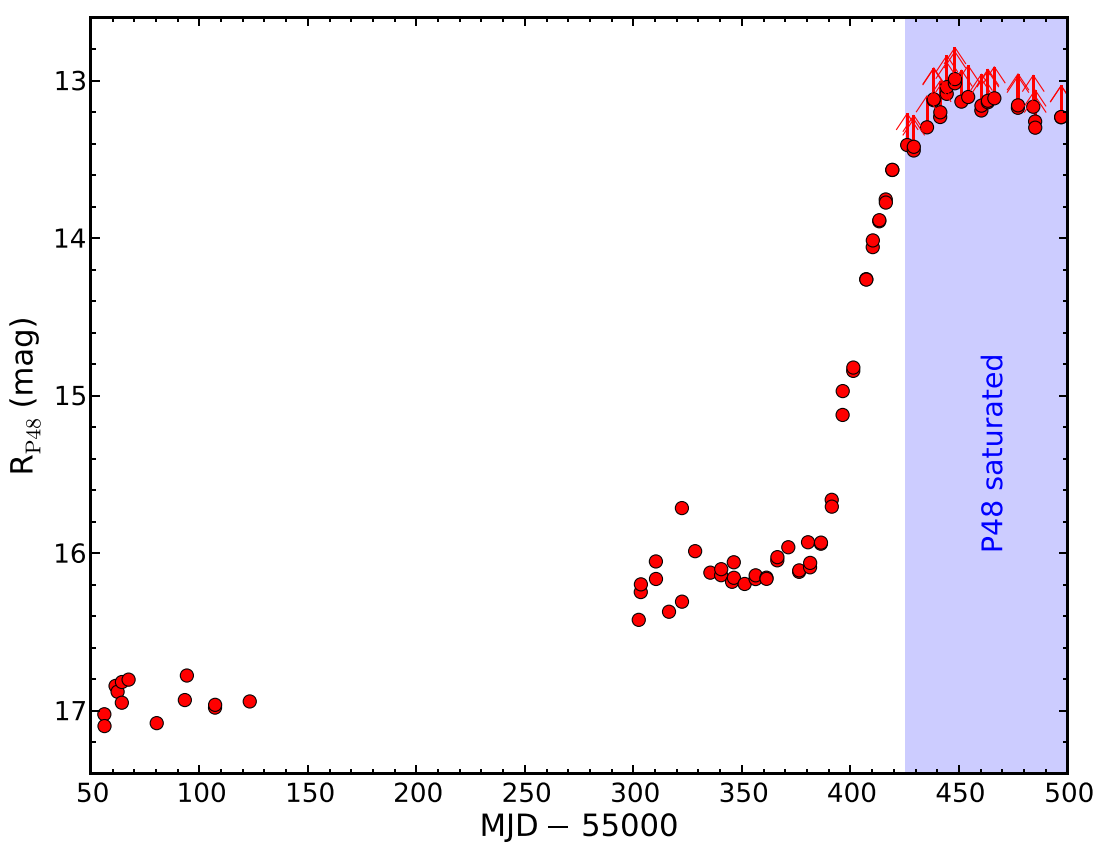

Figure 2. P48 light curve showing the outburst of LkH $\alpha 188$-G4/PTF 10qpf. During the 2009 season, PTF 10qpf was variable with typical $\Delta m \approx 0.2$ mag. The source dramatically increased the rate at which it was brightening around 2010 July 13 (day $\sim 391$ in the figure). P48 saturates on sources brighter than $m_{R_{\mathrm{P} 48}} \approx 13.5$ mag. The shaded region shows all data where PTF 10qpf is saturated; these data represent lower limits to the true brightness of PTF 10qpf during those epochs.

(A color version of this figure is available in the online journal.)

probabilistic classifications of PTF detections (J. S. Bloom et al. 2011, in preparation). These initial classifications are made available to PTF collaboration members via the PTF Follow-up Marshal, which enables visual inspection of current and reference images, precursor PTF light curves, and any subsequent spectroscopy.

The source reported here was detected by the PTF pipeline and automatically assigned the name PTF 10qpf, following internal PTF naming conventions. Differential photometric measurements are made via aperture photometry, with the aperture size scaled to the seeing in each image, using SExtractor (Bertin \& Arnouts 1996), and absolute calibration is relative to the USNO-B1 catalog (Monet et al. 2003) with an uncertainty of $\sim 0.15$ mag. CCD 3 on the P48 camera saturates at 55,000 counts, roughly corresponding to sources with $R \lesssim 13.5 \mathrm{mag}$, given the standard $60 \mathrm{~s}$ exposure time. Thus, in all of our observations following those on 2010 August 14, $\mathrm{LkH} \alpha$ 188G4 is saturated and our measurements represent a lower limit to the true brightness of the source. These limits are reflected in the full P48 light curve of $\mathrm{LkH} \alpha 188-\mathrm{G} 4$, shown in Figure 2, and the photometry is reported in Table 2 . We do not include the systematic $\sim 0.15$ mag calibration uncertainty in this table because this uncertainty represents a constant offset that would be applied to all the data in the same way. Representative images of LkH $\alpha$ 188-G4/PTF 10qpf from the 2009 and 2010 observing seasons are shown in Figure 3.

The PTF also makes use of the robotic Palomar 60 in telescope (P60; Cenko et al. 2006) to obtain multi-filter photometry for source verification and classification purposes. PTF 10qpf was observed with the P60 on 2010 October 14; Figure 4 presents a three-color image constructed from the P60 riz frames. Table 3 contains the individual photometric measurements. The absolute zero-point calibration was done relative to SDSS photometry (Adelman-McCarthy et al. 2008) based on other fields observed by the P60 during the same night with the same filter. The uncertainty in the zero point is computed as the standard deviation of the zero points in all other SDSS fields.

\subsection{Near-infrared Photometry}

Near-infrared observations of PTF 10qpf were conducted with the $1.3 \mathrm{~m}$ Peters Automated Infrared Imaging Telescope (PAIRITEL; Bloom et al. 2006) on Mt. Hopkins, AZ, starting on 2010 September 27. PAIRITEL is a roboticized system using the former 2MASS southern hemisphere survey camera that employs two dichroics to observe simultaneously in the $J, H$, and $K_{s}$ bands. Observations were scheduled and executed via a robotic system. PAIRITEL is operated in a fixed observing mode in which $7.8 \mathrm{~s}$ double-correlated images are created from the difference of a $7.851 \mathrm{~s}$ and a $51 \mathrm{~ms}$ integration taken in rapid succession (see Blake et al. 2008). The standard observing procedure involves taking three image pairs prior to dithering the telescope.

The raw data from these images are reduced using standard IR reduction methods via PAIRITEL PIPELINE III and the flux for all sources is measured via aperture photometry using SExtractor (Bertin \& Arnouts 1996), calibrated against 2MASS. In the $H$ and $K_{s}$ bands, PTF 10qpf saturates the $7.851 \mathrm{~s}$ frames; however, PIPELINE III produces "short-frame" mosaics consisting of reduced, stacked $51 \mathrm{~ms}$ images (see also Bloom et al. 2009). The "short-frame" mosaics contain $>10$ bright 2MASS stars which we use to properly calibrate photometric measurements of PTF $10 q p f$ in these images. PAIRITEL has a systematic uncertainty of $\sim 0.02-0.03$ mag in each of the $J, H$, and $K_{s}$ bands (see Blake et al. 2008; Perley et al. 2010), which, in the case of $\mathrm{LkH} \alpha$ $188-\mathrm{G} 4$, is larger than the statistical error in all three bands. Thus, we add a systematic error of $0.03 \mathrm{mag}$ in quadrature with the statistical uncertainty to determine the total uncertainty in each band. PAIRITEL photometry is reported in Table 4, and a 
Table 2

P48 R-band Photometry of LkH $\alpha$ 188-G4

\begin{tabular}{|c|c|c|}
\hline $\begin{array}{l}\text { Date } \\
\text { (MJD) }\end{array}$ & mag & $\sigma_{\mathrm{mag}}$ \\
\hline 55056.221 & 17.023 & 0.017 \\
\hline 55056.295 & 17.098 & 0.019 \\
\hline 55061.383 & 16.843 & 0.012 \\
\hline 55062.327 & 16.880 & 0.020 \\
\hline 55064.286 & 16.950 & 0.018 \\
\hline 55064.331 & 16.818 & 0.021 \\
\hline 55067.393 & 16.803 & 0.031 \\
\hline 55080.361 & 17.079 & 0.036 \\
\hline 55093.318 & 16.933 & 0.016 \\
\hline 55094.252 & 16.777 & 0.018 \\
\hline 55107.222 & 16.981 & 0.026 \\
\hline 55107.266 & 16.963 & 0.025 \\
\hline 55123.225 & 16.942 & 0.013 \\
\hline 55302.479 & 16.424 & 0.010 \\
\hline 55303.411 & 16.247 & 0.012 \\
\hline 55303.459 & 16.198 & 0.014 \\
\hline 55310.370 & 16.053 & 0.022 \\
\hline 55310.413 & 16.164 & 0.010 \\
\hline 55316.420 & 16.372 & 0.018 \\
\hline 55322.359 & 15.714 & 0.017 \\
\hline 55322.403 & 16.308 & 0.009 \\
\hline 55328.453 & 15.988 & 0.011 \\
\hline 55335.477 & 16.124 & 0.010 \\
\hline 55340.429 & 16.140 & 0.008 \\
\hline 55340.474 & 16.102 & 0.007 \\
\hline 55345.470 & 16.182 & 0.016 \\
\hline 55346.270 & 16.058 & 0.015 \\
\hline 55346.314 & 16.157 & 0.017 \\
\hline 55351.271 & 16.196 & 0.010 \\
\hline 55356.309 & 16.165 & 0.013 \\
\hline 55356.353 & 16.141 & 0.008 \\
\hline 55361.311 & 16.156 & 0.009 \\
\hline 55361.355 & 16.163 & 0.011 \\
\hline 55366.318 & 16.046 & 0.012 \\
\hline 55366.362 & 16.026 & 0.009 \\
\hline 55371.384 & 15.963 & 0.011 \\
\hline 55376.377 & 16.119 & 0.009 \\
\hline 55376.420 & 16.110 & 0.012 \\
\hline 55380.467 & 15.931 & 0.009 \\
\hline 55381.405 & 16.090 & 0.009 \\
\hline 55381.455 & 16.062 & 0.008 \\
\hline 55386.397 & 15.940 & 0.007 \\
\hline 55386.444 & 15.933 & 0.006 \\
\hline 55391.400 & 15.662 & 0.013 \\
\hline 55391.444 & 15.705 & 0.011 \\
\hline 55396.411 & 15.123 & 0.007 \\
\hline 55396.466 & 14.971 & 0.008 \\
\hline 55401.304 & 14.843 & 0.012 \\
\hline 55401.348 & 14.822 & 0.013 \\
\hline 55407.294 & 14.261 & 0.013 \\
\hline 55407.342 & 14.263 & 0.011 \\
\hline 55410.295 & 14.057 & 0.007 \\
\hline 55410.340 & 14.015 & 0.009 \\
\hline 55413.292 & 13.893 & 0.008 \\
\hline 55413.336 & 13.886 & 0.006 \\
\hline 55416.287 & 13.755 & 0.009 \\
\hline 55416.330 & 13.774 & 0.013 \\
\hline 55419.282 & 13.568 & 0.009 \\
\hline 55419.326 & 13.567 & 0.011 \\
\hline 55426.161 & $<13.409$ & $\ldots$ \\
\hline 55429.177 & $<13.444$ & $\ldots$ \\
\hline 55429.220 & $<13.420$ & $\ldots$ \\
\hline 55435.303 & $<13.297$ & $\ldots$ \\
\hline 55438.330 & $<13.124$ & $\ldots$ \\
\hline 55438.413 & $<13.119$ & $\ldots$ \\
\hline
\end{tabular}

Table 2

(Continued)

\begin{tabular}{|c|c|c|}
\hline $\begin{array}{l}\text { Date } \\
\text { (MJD) }\end{array}$ & mag & $\sigma_{\mathrm{mag}}$ \\
\hline 55441.365 & $<13.231$ & $\ldots$ \\
\hline 55441.430 & $<13.201$ & $\ldots$ \\
\hline 55444.390 & $<13.084$ & $\ldots$ \\
\hline 55444.434 & $<13.041$ & $\ldots$ \\
\hline 55448.144 & $<13.017$ & $\ldots$ \\
\hline 55448.189 & $<12.990$ & $\ldots$ \\
\hline 55451.156 & $<13.134$ & $\ldots$ \\
\hline 55454.265 & $<13.104$ & $\ldots$ \\
\hline 55460.339 & $<13.190$ & $\ldots$ \\
\hline 55460.388 & $<13.159$ & $\ldots$ \\
\hline 55463.341 & $<13.137$ & $\ldots$ \\
\hline 55463.386 & $<13.128$ & $\ldots$ \\
\hline 55466.326 & $<13.112$ & $\ldots$ \\
\hline 55477.191 & $<13.173$ & $\ldots$ \\
\hline 55477.234 & $<13.157$ & $\ldots$ \\
\hline 55484.263 & $<13.166$ & $\ldots$ \\
\hline 55485.119 & $<13.260$ & $\ldots$ \\
\hline 55485.163 & $<13.299$ & $\ldots$ \\
\hline 55497.098 & $<13.233$ & $\ldots$ \\
\hline 55497.141 & $<13.232$ & $\ldots$ \\
\hline
\end{tabular}

Table 3

P60 Observations of $\mathrm{LkH} \alpha$ 188-G4

\begin{tabular}{lccc}
\hline \hline $\begin{array}{l}\text { Date } \\
(\mathrm{MJD})\end{array}$ & Filter & $\begin{array}{l}\text { mag } \\
(\mathrm{AB})\end{array}$ & $\sigma_{\text {mag }}$ \\
\hline 55483.127 & $r$ & 12.948 & 0.039 \\
55483.127 & $i$ & 12.207 & 0.027 \\
55483.128 & $z$ & 11.623 & 0.041 \\
\hline
\end{tabular}

Table 4

PAIRITEL Observations of $\mathrm{LkH} \alpha 188-\mathrm{G} 4$

\begin{tabular}{lccc}
\hline \hline $\begin{array}{l}t_{\text {mid }}{ }^{\mathrm{a}} \\
(\mathrm{MJD})\end{array}$ & $\begin{array}{c}J \text { mag } \\
(\mathrm{Vega})\end{array}$ & $\begin{array}{c}H \text { mag } \\
(\text { Vega })\end{array}$ & $\begin{array}{c}K_{s} \text { mag } \\
(\text { Vega })\end{array}$ \\
\hline 55466.137 & $10.04 \pm 0.03$ & $9.14 \pm 0.03$ & $8.65 \pm 0.03$ \\
55468.145 & $9.99 \pm 0.03$ & $9.06 \pm 0.04$ & $8.64 \pm 0.04$ \\
55469.148 & $10.04 \pm 0.03$ & $9.07 \pm 0.03$ & $8.70 \pm 0.03$ \\
55479.109 & $10.11 \pm 0.03$ & $9.11 \pm 0.03$ & $8.63 \pm 0.04$ \\
55504.164 & $10.20 \pm 0.03$ & $9.24 \pm 0.03$ & $8.74 \pm 0.03$ \\
55513.195 & $10.29 \pm 0.03$ & $9.34 \pm 0.03$ & $8.79 \pm 0.03$ \\
55518.168 & $10.32 \pm 0.03$ & $9.34 \pm 0.04$ & $8.84 \pm 0.03$ \\
55527.117 & $10.35 \pm 0.03$ & $9.40 \pm 0.03$ & $8.89 \pm 0.03$ \\
55531.145 & $10.40 \pm 0.03$ & $9.44 \pm 0.03$ & $8.97 \pm 0.03$ \\
55543.066 & $10.45 \pm 0.03$ & $9.48 \pm 0.04$ & $9.06 \pm 0.04$
\end{tabular}

Note. ${ }^{\text {a }}$ Midpoint between the first and last exposures in a single stacked image.

$J, H$, and $K_{s}$ band false-color image of PTF 10qpf is shown in Figure 5.

\subsection{Optical Spectroscopy \\ 3.3.1. Lick Spectroscopy}

Low-resolution spectra of PTF 10qpf were obtained on 2010 September 16 and 2010 November 2 with the Kast spectrograph on the Lick 3 m Shane telescope (Miller \& Stone 1993). The spectra were reduced and calibrated using standard procedures (e.g., Matheson et al. 2000). For the September 16 spectrum, flux calibration for the red arm of the Kast spectrograph was performed using a standard star observed at high airmass, 


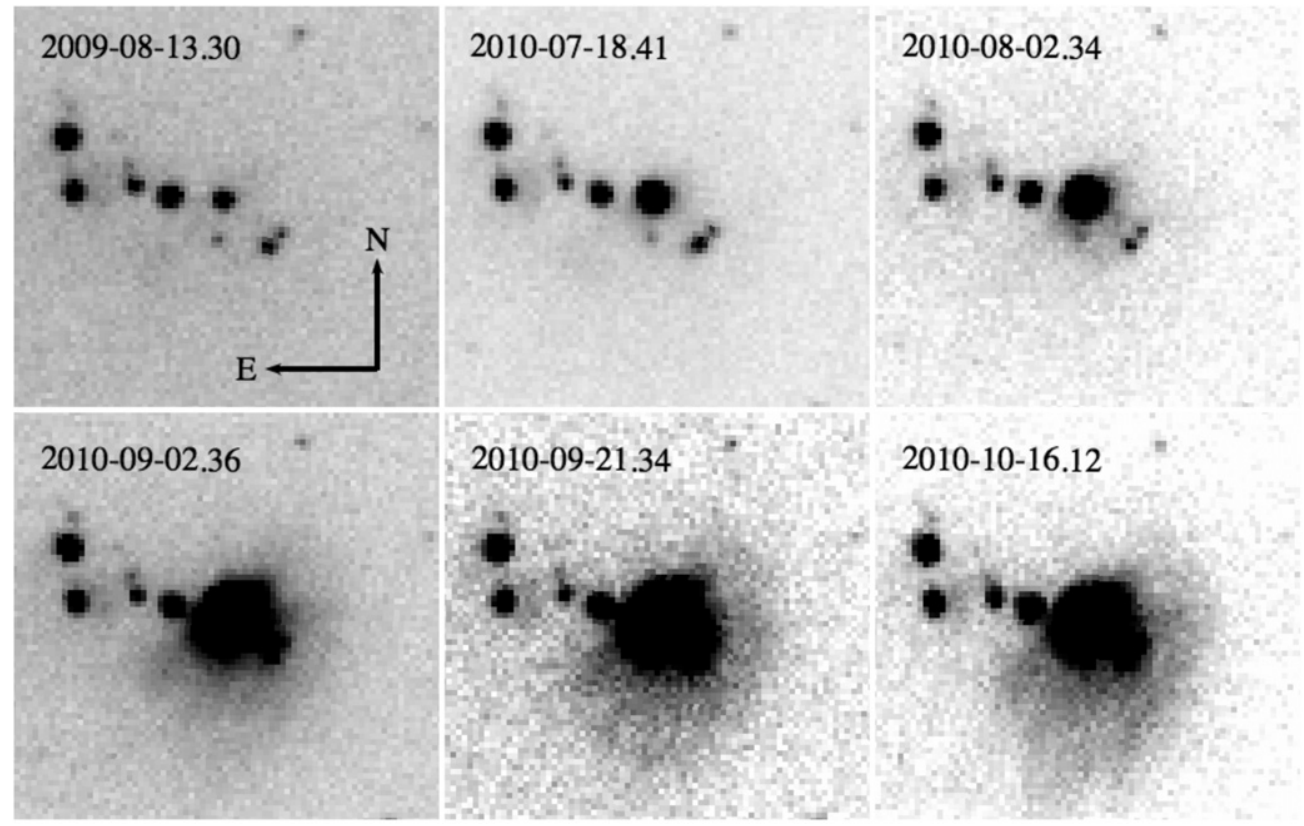

Figure 3. P48 R-band images of PTF 10qpf showing the emergence of the optical reflection nebula. Each image is centered on PTF 10qpf and is $75^{\prime \prime}$ on a side. As with many other FU Orionis-like variables, the outburst of PTF 10qpf has created an asymmetric reflection nebula. Note that PTF 10qpf is not located in the center of the reflection nebula which extends to the southwest of the pre-outburst point source. All images are registered to the same frame with north up and east to the left. All dates are UT.

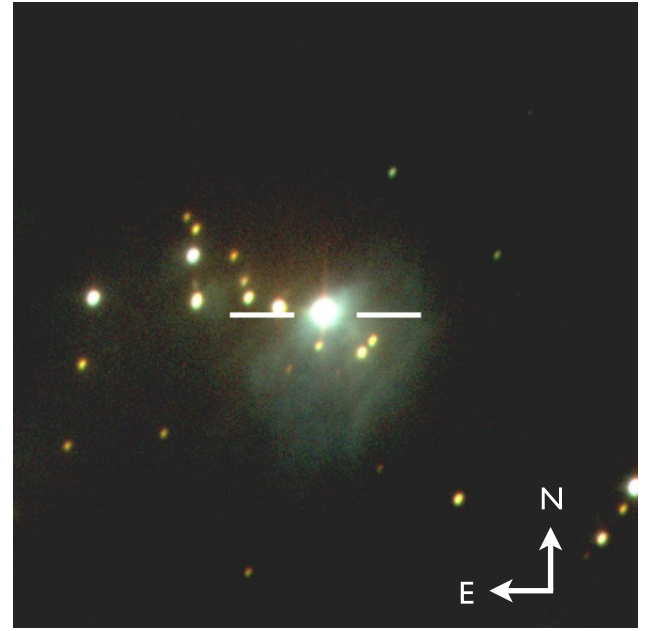

Figure 4. False-color riz image of PTF 10qpf during the 2010 eruption. The image is 2.2 on a side with north up and east to the left. PTF 10qpf is highlighted with crosshairs. Note the bright reflection nebula to the southwest of PTF 10qpf; this nebula was not present prior to the 2010 eruption.

(A color version of this figure is available in the online journal.)

whereas PTF 10qpf was observed at low airmass; thus, the absolute flux calibration is somewhat uncertain in the red portion of the optical spectrum. Furthermore, the noisy features near $\sim 9300 \AA$ are likely the result of an imperfect telluric correction and not astrophysical. Since conditions were not photometric on the night of November 2, the absolute flux calibration is again somewhat uncertain. Nevertheless, on both nights we observed PTF 10qpf with the slit placed at the parallactic angle, so the relative spectral shapes should be accurate. The full low-resolution spectra of PTF 10qpf are shown in Figure 6, while all of our spectroscopic observations are logged in Table 5.

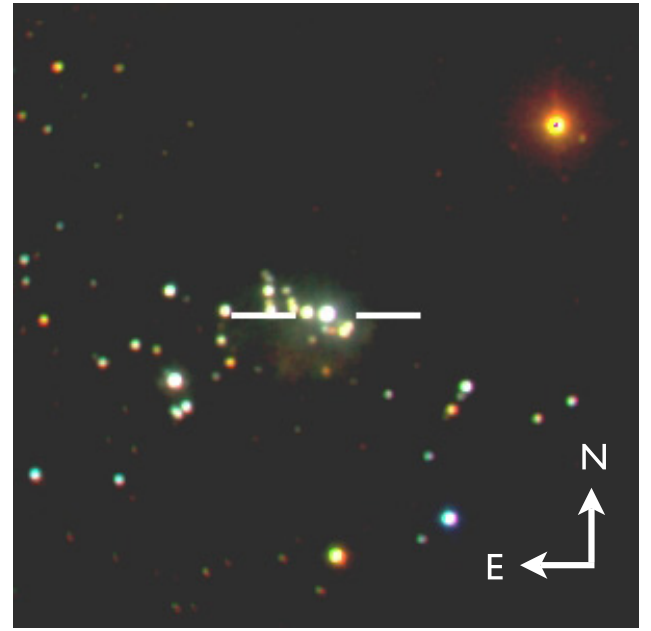

Figure 5. False-color $J H K_{s}$ image of PTF 10qpf during the 2010 eruption. The NIR reflection nebula is less prominent than the optical nebula, but still visible to the southwest of PTF 10qpf. The image is $5^{\prime}$ on a side with north up and east to the left. PTF 10qpf is highlighted with crosshairs.

(A color version of this figure is available in the online journal.)

Table 5

Log of Spectroscopic Observations

\begin{tabular}{lccc}
\hline \hline UT Date & Telescope/ & Exposure & $\lambda$ \\
& Instrument & $(\mathrm{s})$ & $(\AA)$ \\
\hline $2010-09-16.24$ & Shane 3 m/Kast & 600 & $3400-10300$ \\
$2010-09-23.24$ & Hale 5 m/TripleSpec & 720 & $9400-24,600$ \\
$2010-09-25.30$ & Keck I 10 m/HIRES & 560 & $3640-7990$ \\
$2010-11-02.21$ & Shane 3 m/Kast & 1200 & $3400-10300$ \\
\hline
\end{tabular}

\subsubsection{High-resolution Spectroscopy}

PTF 10qpf was observed on 2010 September 25 with the Keck I telescope and HIRES spectrometer (Vogt et al. 1994) using the 


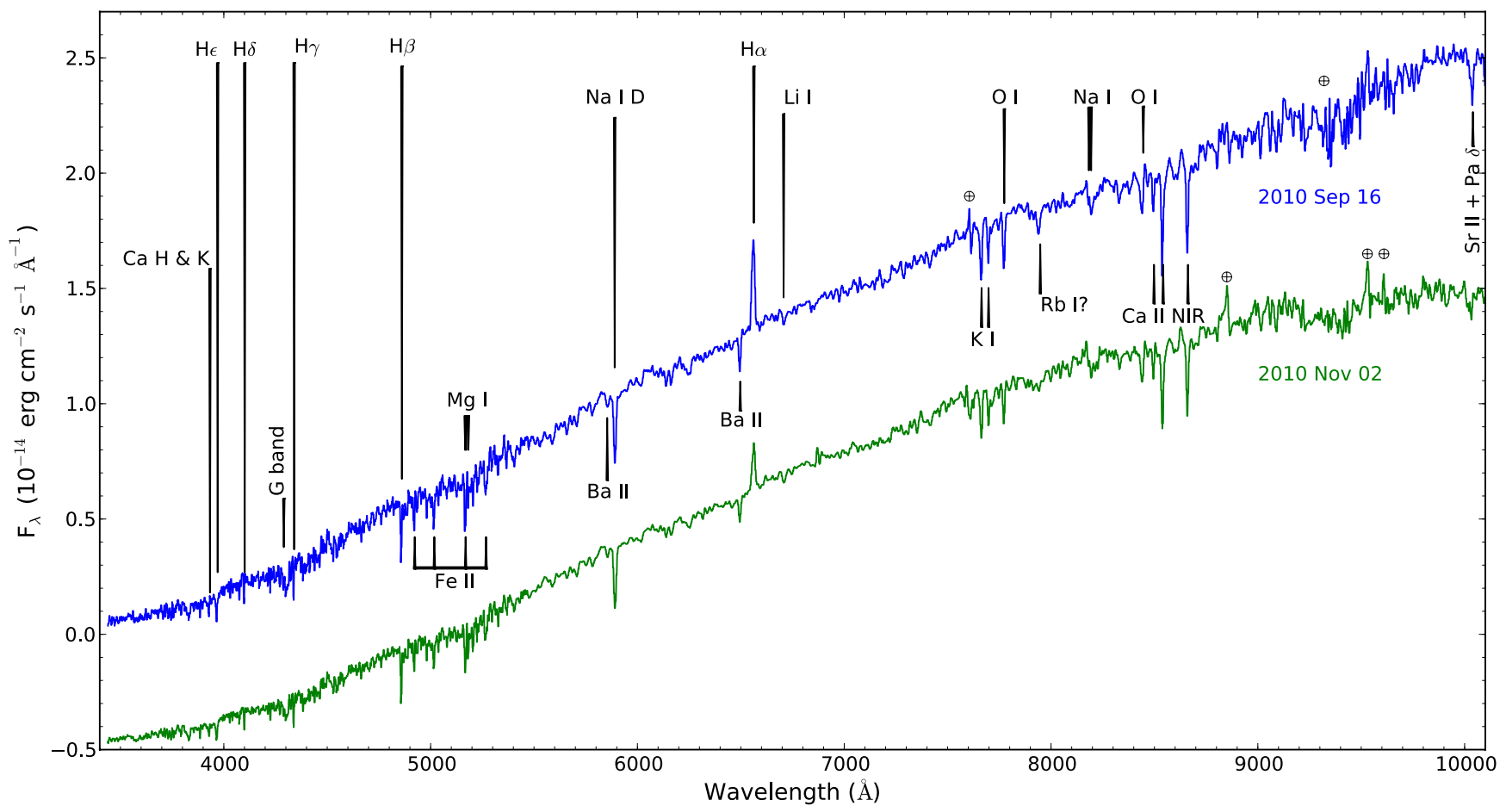

Figure 6. Low-resolution spectra of PTF 10qpf. Prominent absorption lines are marked. H $\alpha$ is the only line seen in emission, and it appears to have decreased in strength between our two spectra. Residuals left following an imperfect removal of telluric absorption have been marked with a $\oplus$, while the noisy features around $\sim 9300 \AA$ are also likely telluric (see the text). The spectrum from 2010 November 2 has been shifted down by 0.5 for clarity.

(A color version of this figure is available in the online journal.)

red cross disperser and the standard settings of the California Planet Survey (CPS; Howard et al. 2010; Johnson et al. 2010). The $\mathrm{C} 2$ decker was employed, projecting to 0'.86 on the sky and providing a resolution $R=\lambda / \Delta \lambda=55,000$ at $5500 \AA$. We took advantage of the standard CPS reduction pipeline, which includes flat-fielding, scattered-light subtraction, order tracing, cosmic ray rejection, and spectrum extraction. After extraction, each order is sky-subtracted and summed in the cross-dispersion direction to form the final one-dimensional spectrum. The exposure time of $560 \mathrm{~s}$ resulted in a signal-tonoise ratio $(\mathrm{S} / \mathrm{N})$ of $\sim 20$ at $7400 \AA$.

\subsubsection{Near-infrared Spectroscopy}

A NIR spectrum of PTF 10qpf was obtained with the TripleSpec spectrograph on the Palomar $5 \mathrm{~m}$ Hale telescope (Herter et al. 2008) on 2010 September 23. TripleSpec has no moving parts and simultaneously acquires five cross-dispersed orders covering $1.0-2.4 \mu \mathrm{m}$ at $R \approx 2700$.

The spectra were reduced with an IDL-based data reduction pipeline developed by P. Muirhead. ${ }^{12}$ To facilitate the subtraction of the sky and background emission of the total signal, the observations were obtained using an ABBA dither pattern along the slit. Each sky-subtracted exposure was then divided by a normalized flat field, wavelength-calibrated, and optimally extracted (Horne 1986). The spectra were flux-calibrated and corrected for telluric absorption using observations of an A0V star at a similar airmass with the IDL-based code xtellcor_general by Vacca et al. (2003). The full TripleSpec spectrum is shown in Figure 7.

\footnotetext{
12 http://www.astro.cornell.edu/ muirhead/\#Downloads.
}

\section{ANALYSIS}

\subsection{Image Morphology and Photometric Analysis}

The large-amplitude eruption ( $\sim \mathrm{mag}$ ) of $\mathrm{LkH} \alpha$ 188-G4/ PTF 10qpf qualitatively matches the defining characteristic of FU Orionis eruptions. Such an eruption, however, is not sufficient to prove that a YSO is in an FU Orionis-like state. PTF $10 \mathrm{nvg}$, for example, brightened by $\sim 6$ mag over the course of a year, yet shows little resemblance to FU Ori-like stars (Covey et al. 2011). Spectroscopy over a broad wavelength range (see below) is required to conclusively classify a source as an FU Orionis-like star.

Over the course of our P48 observations, PTF 10qpf brightened by $\sim 1$ mag from early 2009 August to early 2010 August, before suddenly brightening by $\sim 3$ mag over a $\sim 2$ month period (see Figure 2). Between the start of our observations on 2009 August 13 and 2010 July 9, the initial slow rising phase, PTF 10 qpf rose by an average of $\sim 0.003 \mathrm{mag} \mathrm{day}^{-1}$, while between 2010 July 9 and August 11, during the rapid rising phase, PTF 10 qpf rose by an average of $\sim 0.07 \mathrm{mag} \mathrm{day}^{-1}$.

After 2010 August 11, PTF 10qpf is saturated in the P48 images and we cannot constrain the rate at which it is increasing; however, Semkov \& Peneva (2010b) report that it reached peak brightness on 2010 August 24 with $R=12.79 \mathrm{mag}$. Despite the rather rapid rise at the beginning of the PTF 10qpf optical outburst, the overall light curve is remarkably smooth. The P48 light curve for PTF 10qpf shows fluctuations at the $\sim 0.2 \mathrm{mag}$ level about both the pre-outburst slow rise and the sharp rise of the outburst. The long-term trend with low scatter observed prior to 2010 July is somewhat unusual for a standard CTTS, whose year-to-year variability is typically significantly smaller than the intra-year variability (Grankin et al. 2007). Indeed, the IPHAS photometry of $\mathrm{LkH} \alpha 188-\mathrm{G} 4$ captures variability that 


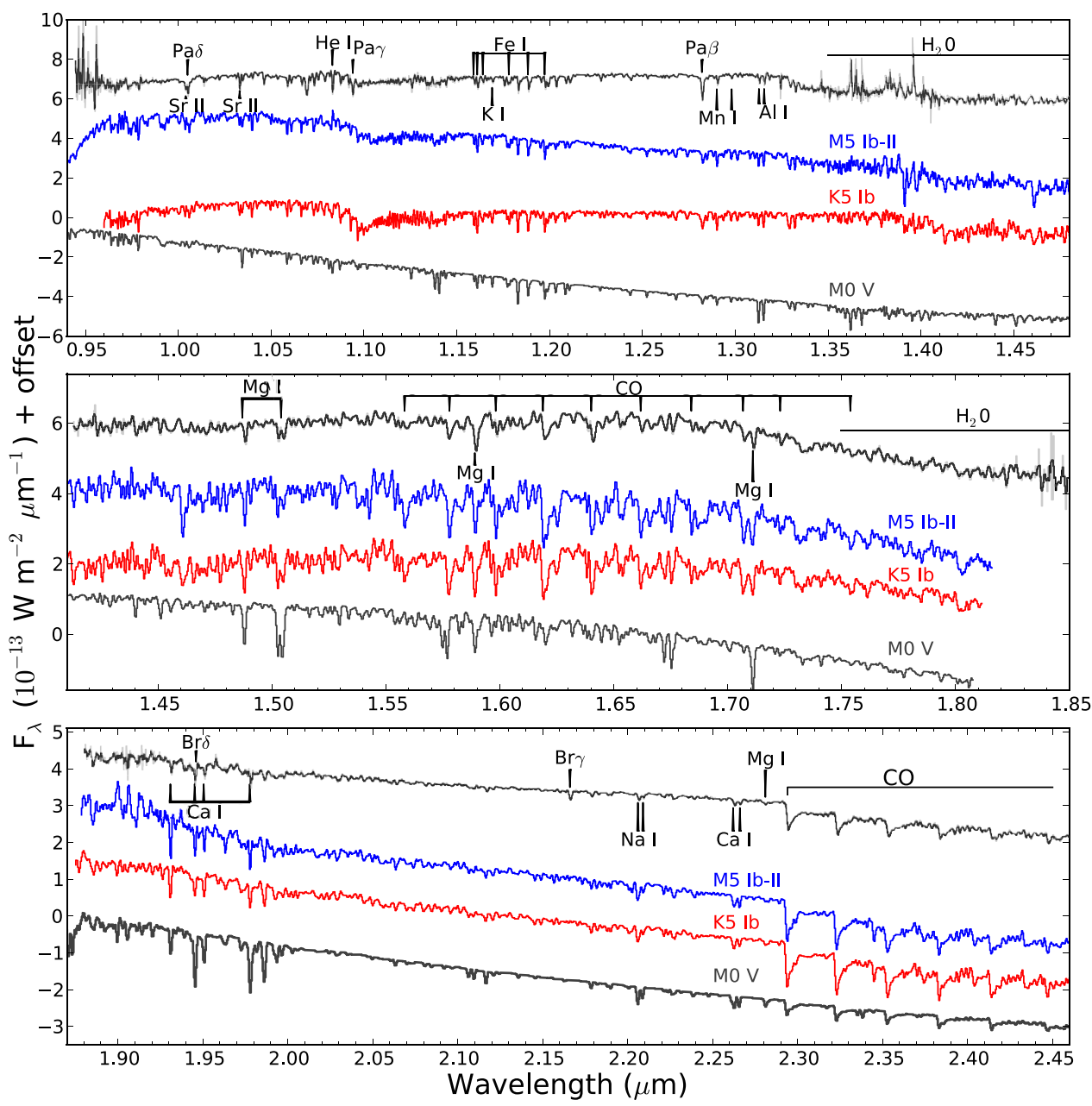

Figure 7. TripleSpec NIR spectrum of PTF 10qpf showing from top to bottom the $Y J-, H$-, and $K$-band spectra. Prominent absorption features in the outburst spectrum are identified, while the spectrum shows no clear emission lines. For comparison, the spectra of M5 Ib-II and K5 Ib supergiants, and an M0 V dwarf (from Rayner et al. 2009), are also shown. The muted features of PTF 10qpf relative to the M0 V star show that the NIR emission is from a low surface gravity environment. PTF 10qpf exhibits strong $2.3 \mu \mathrm{m} \mathrm{CO}$ absorption and $\sim 1.4$ and $1.9 \mu \mathrm{m} \mathrm{H}_{2} \mathrm{O}$ absorption, similar to other FU Orionis-like sources.

(A color version of this figure is available in the online journal.)

is more characteristic of CTTSs: variability at the $\sim 0.3 \mathrm{mag}$ level was observed over the course of 1 month in 2003, with a follow-up observation two years later falling within the same range of magnitudes as observed in 2003. This suggests that the slow rise $\mathrm{LkH} \alpha$ 188-G4 demonstrated in 2009 was a prelude to the 2010 outburst, rather than simply standard CTTS variability.

A new reflection nebula, which was not present in 2009, can now clearly be seen around PTF 10qpf (Figure 3). Our regular observations over the course of the rise of PTF 10qpf allow us to constrain the appearance of the nebula, similar to the analysis of V1647 Ori/McNeil's Nebula by Briceño et al. (2004). In the P48 subtraction images produced as part of the PTF reduction pipeline, the residual is asymmetric about the point-source centroid on 2010 August 2. The dust responsible for the reflection nebula is offset to the southwest of PTF 10qpf, and the asymmetry in the subtraction image is the result of this dust scattering light into our line of sight. We measure $R \approx 14 \mathrm{mag}$ for PTF 10qpf on 2010 August 2 . The nebula may be present as early as 2010 July 19 , however: the faint nebula is difficult to distinguish from the wings of the central star's point-spread function. The optical reflection nebula (Figure 4) has an apparent size of $\sim 40^{\prime \prime}$, which at a distance of $\sim 600 \mathrm{pc}$ corresponds to $\sim 24,000 \mathrm{AU}$.
In the NIR, we have less information than in the optical. Our observations suggest a flat light curve in the $J, H$, and $K_{s}$ bands starting on 2010 September 27. Our NIR photometric measurements are consistent with those presented by Leoni et al. (2010), and we confirm that PTF 10qpf has brightened by 3.25, 3.07 , and $2.81 \mathrm{mag}$ in the $J, H$, and $K_{s}$ bands, respectively, since 2MASS observations were obtained on 2000 June 10 . The NIR measurements presented by Cohen \& Kuhi (1979) are $\sim 1.1 \mathrm{mag}$ brighter than those of 2MASS, which may be the result of a larger beam size for the Cohen \& Kuhi (1979) measurements. FU Orionis stars are frequently associated with NIR reflection nebulae (Connelley et al. 2007; Connelley \& Greene 2010), and PTF 10qpf is no exception (see Figure 5). The NIR nebula is similar in size to the nebula seen in the optical.

The outburst SED is shown in Figure 1, and it clearly demonstrates that PTF 10qpf is bluer in the optical/NIR than it was prior to the 2010 outburst. We have limited spectral coverage during the outburst; in particular, we lack any information on how the emission has changed in the MIR. Direct integration of the optical/NIR outburst SED results in a lower limit to the luminosity of at least $4 L_{\odot}$. Assuming the same bolometric correction to the $H$ band before and after the outburst results in an outburst luminosity of $L_{\text {outburst }} \approx 12 L_{\odot}$. This luminosity 

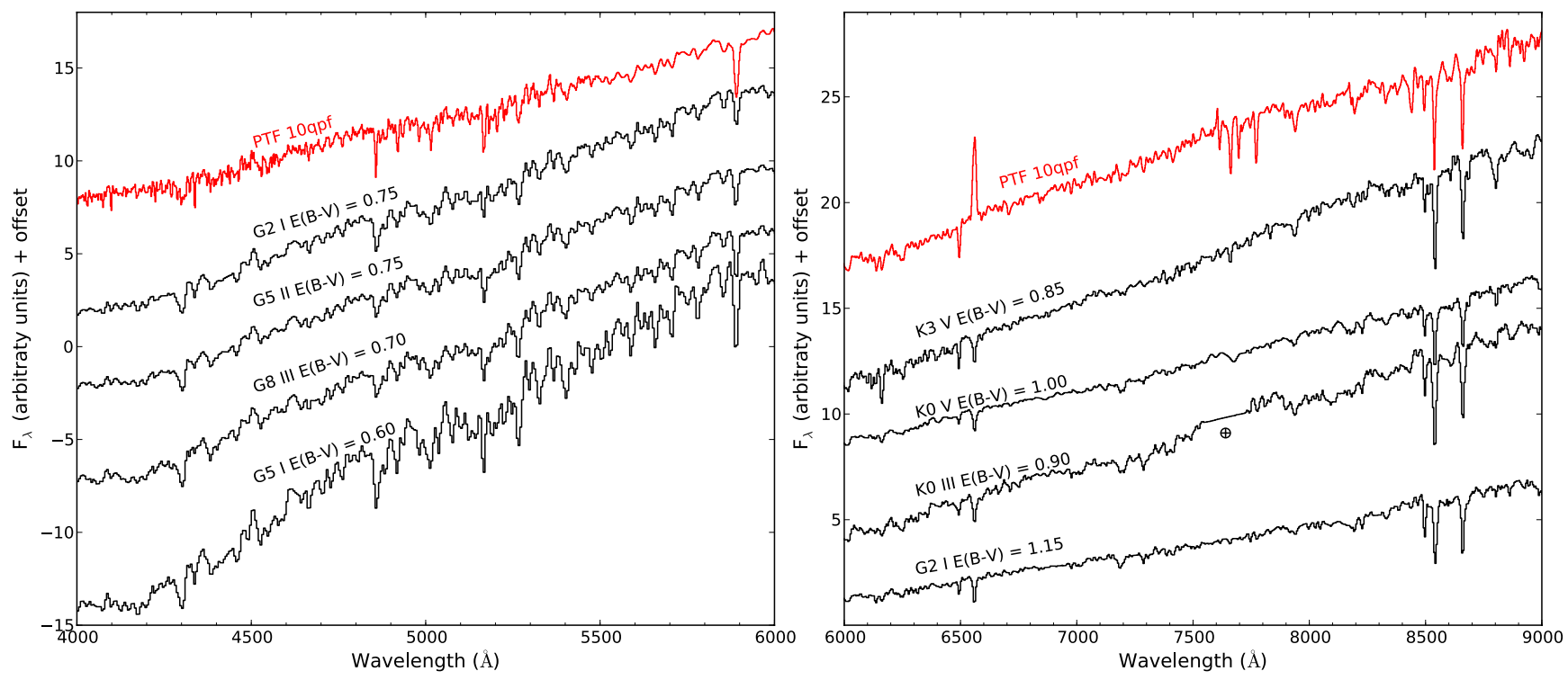

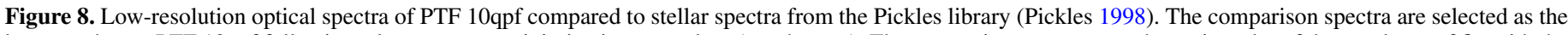

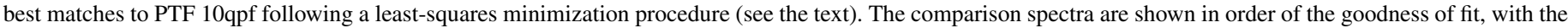
best match on top. The reddening values were used as a proxy for removing the overall continuum shape.

(A color version of this figure is available in the online journal.)

places PTF 10qpf at the bottom of the luminosity scale for FU Ori outbursts, and more than an order of magnitude fainter than FU Ori itself (Hartmann \& Kenyon 1996).

\subsection{Spectroscopic Analysis}

Optical spectra of FU Orionis variables share a few defining properties: a late $\mathrm{F}$ or $\mathrm{G}$ supergiant photosphere, an absorptiondominated spectrum with only $\mathrm{H} \alpha$ typically seen in emission, and broad $\mathrm{P}$ Cygni absorption with representative velocities of a few hundred $\mathrm{km} \mathrm{s}^{-1}$. Figure 6 shows that PTF 10qpf exhibits these general properties. Figure 9 illustrates our HIRES spectra covering three Balmer transitions. Each of these lines exhibits a strong $\mathrm{P}$ Cygni absorption profile, with absorption minima around $-40 \mathrm{~km} \mathrm{~s}^{-1}$. $\mathrm{H} \alpha$ is the only line having an emission component, and this emission moves the absorption minimum slightly blueward of the other Balmer lines. The absorption extends to $\lesssim-200 \mathrm{~km} \mathrm{~s}^{-1}$, suggesting in the classical interpretation that PTF 10qpf is driving a strong outflow (see also Figure 9).

In the discussion below, all velocities have been transformed to the local standard of rest (LSR). To our knowledge, a precise radial velocity measurement for $\mathrm{LkH} \alpha 188-\mathrm{G} 4$ has not been made. Therefore, we adopt a rest velocity of $V_{\mathrm{LSR}} \approx 1.6 \mathrm{~km} \mathrm{~s}^{-1}$, consistent with ${ }^{13} \mathrm{CO}$ observations of the surrounding molecular gas (Dobashi et al. 1994).

\subsubsection{Low-resolution Optical Spectroscopy}

At low resolution, PTF 10qpf exhibits little evolution between our two Kast spectra taken 40 day apart (see Figure 6). The strength of the absorption features remains constant to within the uncertainties, while the only major change is in the emission flux from $\mathrm{H} \alpha$. During this 40 day period, the $\mathrm{H} \alpha$ flux has decreased from $\sim 2.8 \times 10^{30} \mathrm{erg} \mathrm{s}^{-1}$ to $\sim 1.5 \times 10^{30} \mathrm{erg} \mathrm{s}^{-1}$. Observing at the parallactic angle allows us to accurately measure the change in the relative color during the 40 day gap between observations. Given the typical uncertainties associated with spectrophotometric measurements with the Kast spectrograph (J. M. Silverman et al. 2011, in preparation), we find that PTF 10qpf does not show significant evidence for a change in color between our two observations.

To constrain the optical spectral type of PTF 10qpf, we use a least-squares minimization procedure to find the best match between the low-resolution Kast spectrum and the low-resolution stellar spectra provided in the Pickles Stellar Spectral Flux Library (Pickles 1998). The spectral type of FU Orionis variables is known to vary as a function of wavelength (Herbig 1977); thus, we divide the spectra into two sections, 4000-6000 A and 6000-9000 A. Below $4000 \AA$, the $S / N$ is low in our Kast spectrum, while above $\sim 9000 \AA$ there is an uncertain telluric correction which may add artificial features. Next, we redden each of the Pickles star spectra in increments of $0.05 \mathrm{mag}$ from $E(B-V)=0.00$ to $2.25 \mathrm{mag}$ with $R_{V}=3.1$. We mask the region around $\mathrm{H} \alpha$, since emission lines are not expected in normal stellar spectra. We then perform a least-squares fit of the reddened library spectra with our low-resolution spectrum of $\mathrm{LkH} \alpha 188-\mathrm{G} 4$, where the only free parameter is an overall normalization constant.

We illustrate the results of this procedure in Figure 8, where we include the low-resolution spectrum of PTF 10qpf along with the four best matches from our least-squares minimization. The stellar spectra are shown in the order of the quality of the fit, with the best fit on top and the fourth-best fit on the bottom. The adopted reddening has little physical meaning given that the observed flux is not emanating from a stellar photosphere having the standard single-temperature SED; this portion of the procedure merely serves as an automated process to vary the slope of the continuum, which is necessary given that PTF 10qpf has a heavily extinguished spectrum.

The Pickles library is incomplete-it does not include every luminosity class for every spectral type. For this reason, we emphasize that our procedure is only illustrative; it does not provide a definitive spectral type for PTF 10qpf in either the blue or the red portion of the optical spectrum. Nevertheless, 

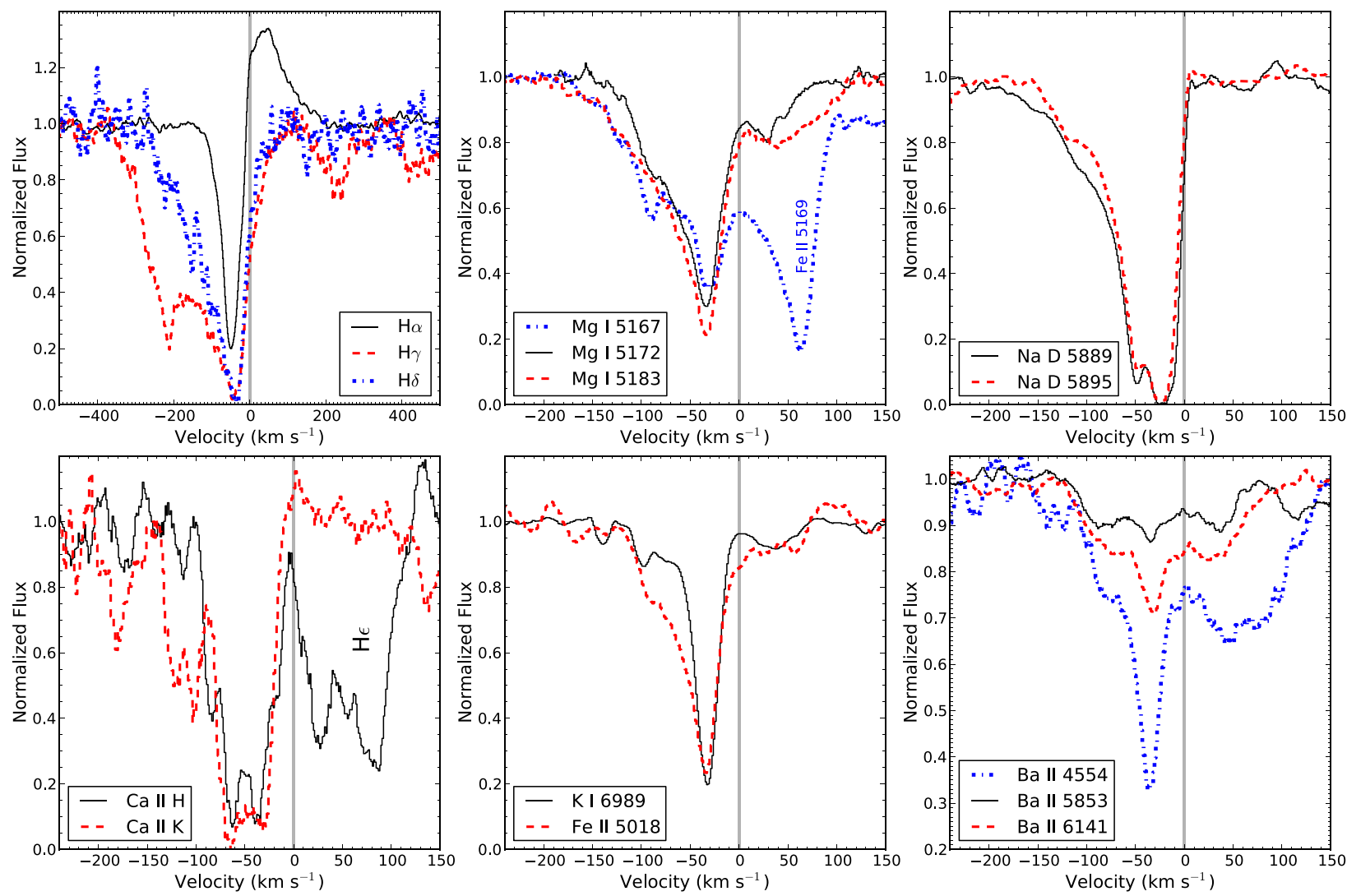

Figure 9. Keck HIRES high-resolution profiles of several prominent features showing strong blueshifted absorption, indicative of outflowing material. Top left: the Balmer series for PTF 10qpf. H $\beta$ fell in a gap between echelle orders for our observing setup and thus is not displayed. PTF 10qpf exhibits strong P Cygni absorption in the other Balmer lines with outflow speeds of $\sim 100-300 \mathrm{~km} \mathrm{~s}^{-1}$. Top center: profiles of the Mg I triplet at 5167, 5172, and $5183 \AA$ A. The lines show strong asymmetries, tracing the kinematics of the wind from PTF 10qpf. Fe II $\lambda 5169$ is present at about $+60 \mathrm{~km} \mathrm{~s}^{-1}$ relative to $\mathrm{Mg}$ I $\lambda 5167$. Top right: absorption profiles of the Na I D doublet showing similar kinematics as the $\mathrm{Mg}$ I lines. Bottom left: absorption profiles of $\mathrm{Ca}$ II $\mathrm{H}$ and $\mathrm{K}$. $\mathrm{H} \epsilon$ is also present, offset from Ca $\mathrm{H} \mathrm{by} \sim 100 \mathrm{~km} \mathrm{~s}{ }^{-1}$. Bottom center: absorption profiles of Fe II $\lambda 5018$ and $\mathrm{K}_{\mathrm{I}} \lambda 6989$. Bottom right: absorption profiles of Ba II $\lambda \lambda 4554,5853$, and 6141 . These lines show narrow absorption, FWHM $\approx 20 \mathrm{~km} \mathrm{~s}^{-1}$, superposed on broader, shallower profiles extending $\pm \sim 60 \mathrm{~km} \mathrm{~s}^{-1}$ from the line center. For clarity, all spectra have been smoothed with a Savitzky-Golay filter, which is similar to a running mean (Savitzky \& Golay 1964). Velocities are given relative to the LSR, and the vertical gray line indicates the rest velocity of PTF $10 \mathrm{qpf}, V_{\mathrm{LSR}} \approx 1.6 \mathrm{~km} \mathrm{~s}^{-1}$ (see the text).

(A color version of this figure is available in the online journal.)

a few trends are readily apparent from Figure 8. In the blue portion of the optical, PTF 10qpf most closely resembles the spectra of $\mathrm{G}$ supergiants and $\mathrm{G}$ giants. In the red portion of the optical, the spectrum resembles that of $\mathrm{G}$ and $\mathrm{K}$ stars, with no real hint regarding the luminosity class. The adopted values of the reddening are significantly different between the blue and the red, but again we remind the reader that this reddening is only a device with which to match the intrinsic shape of the continuum. Typically, the $\operatorname{Mg}$ I $\lambda 5172$ triplet is used to determine the luminosity class of G-type stars, but our highresolution spectra show that these lines are tracing an outflow (see Figure 9) and thus they are unsuitable for providing a luminosity class. Despite this, the procedure does demonstrate that PTF 10qpf is similar to G giants/supergiants in the blue portion of the optical, consistent with observations of other FU Orionis variables (Hartmann \& Kenyon 1996). Additionally, as we will show below, the spectrum shifts to later types (K stars in this case) with increasing wavelength, as is also observed in FU Orionis variables (Herbig 1977).

\subsubsection{High-resolution Optical Spectra}

The HIRES spectrum of PTF 10qpf is dominated by broadened stellar-like absorption features. The spectral lines are consistent with those of a comparison G2 I star and inconsistent with spectral types later than early $\mathrm{K}$. We note, however, that the diagnostics of $\mathrm{G}$ versus $\mathrm{K}$ types are subtle and would become blurred with the large broadening of the PTF 10qpf lines. The line broadening is similar to, but perhaps slightly less than, the $60-100 \mathrm{~km} \mathrm{~s}^{-1}$ broadening of V1057 Cyg, and significantly more than the $30-40 \mathrm{~km} \mathrm{~s}^{-1}$ of V1515 Cyg in which some hints of a K-type spectrum can be seen at redder optical wavelengths. A detailed comparison of PTF 10qpf to these two canonical FU Ori objects reveals that the spectrum of PTF 10qpf is similar to both in terms of the relative strength of the common absorption features. However, we note that each object has unique and notable spectroscopic attributes.

Several lines have clear kinematic signatures of winds, specifically Ca II $\mathrm{H}$ and $\mathrm{K}, \mathrm{Fe}$ II $\lambda 5018$, the $\mathrm{Mg}$ I $\lambda \lambda 5167,5172$, 5183 triplet, the Na I D lines, $\mathrm{H} \alpha, \mathrm{H} \gamma, \mathrm{H} \delta(\mathrm{H} \beta$ falls in a gap between the orders in our HIRES setting), and the $\mathrm{K}_{\mathrm{I}} \lambda 7699$ line (its doublet partner at $7665 \AA$ also falls in a gap between orders). The morphology of these lines is illustrated in Figure 9. Notably, the blueshifted absorption troughs are narrower in our early-stage spectrum of PTF 10qpf than exhibited in modern spectra of V1515 Cyg and V1057 Cyg, by at least a factor of two. Although LkH $\alpha$ 188-G4 exhibited [O I] $\lambda 6300$ emission in the Cohen \& Kuhi (1979) spectrum, it is not apparent postoutburst, even at high spectral resolution, nor do we see other 


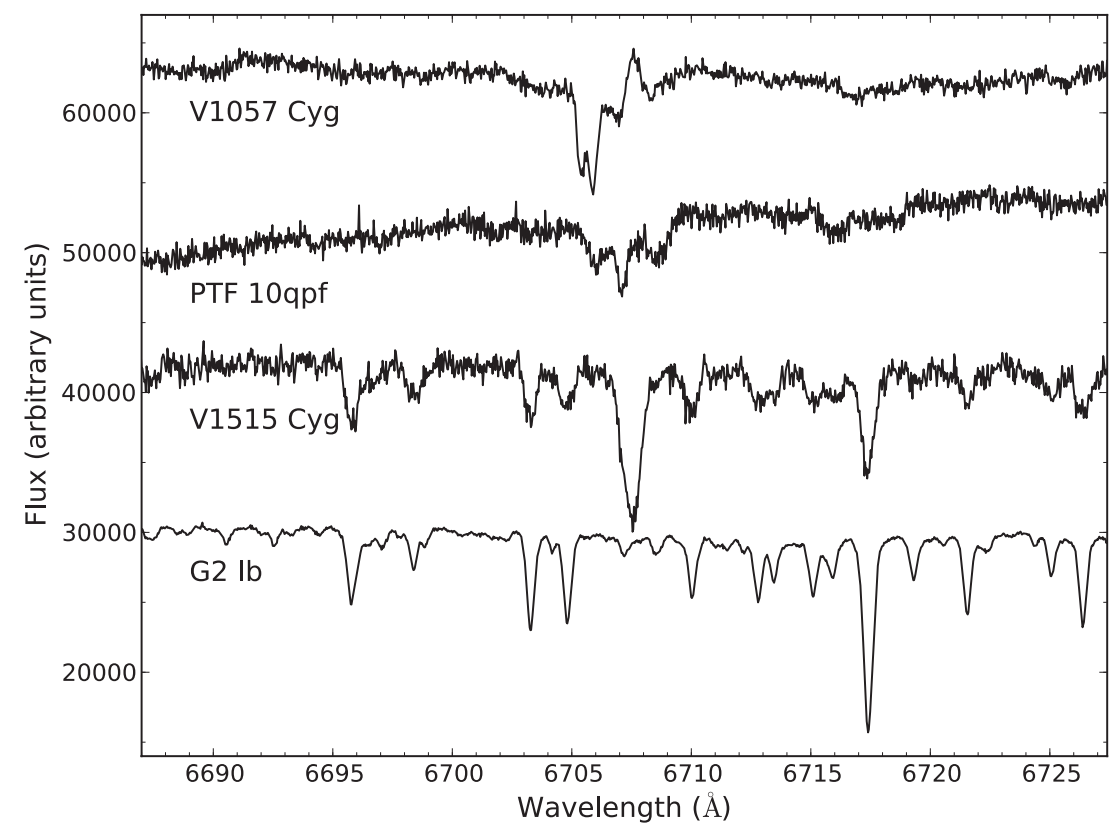

Figure 10. Keck HIRES high-resolution spectra of the Li I $\lambda 6706$ and Ca I $\lambda 6717$ region of PTF 10qpf. For comparison, we also show high-resolution spectra of V1057 Cyg, V1515 Cyg, and a G2 Ib spectral standard, all from our unpublished spectral database. In particular, note the strong Li absorption present in PTF 10qpf and the two canonical FU Orionis objects. The Li line in both V1057 Cyg and PTF 10qpf traces an unusual kinematic structure, possibly similar to that seen in Ba II (see Figure 9).

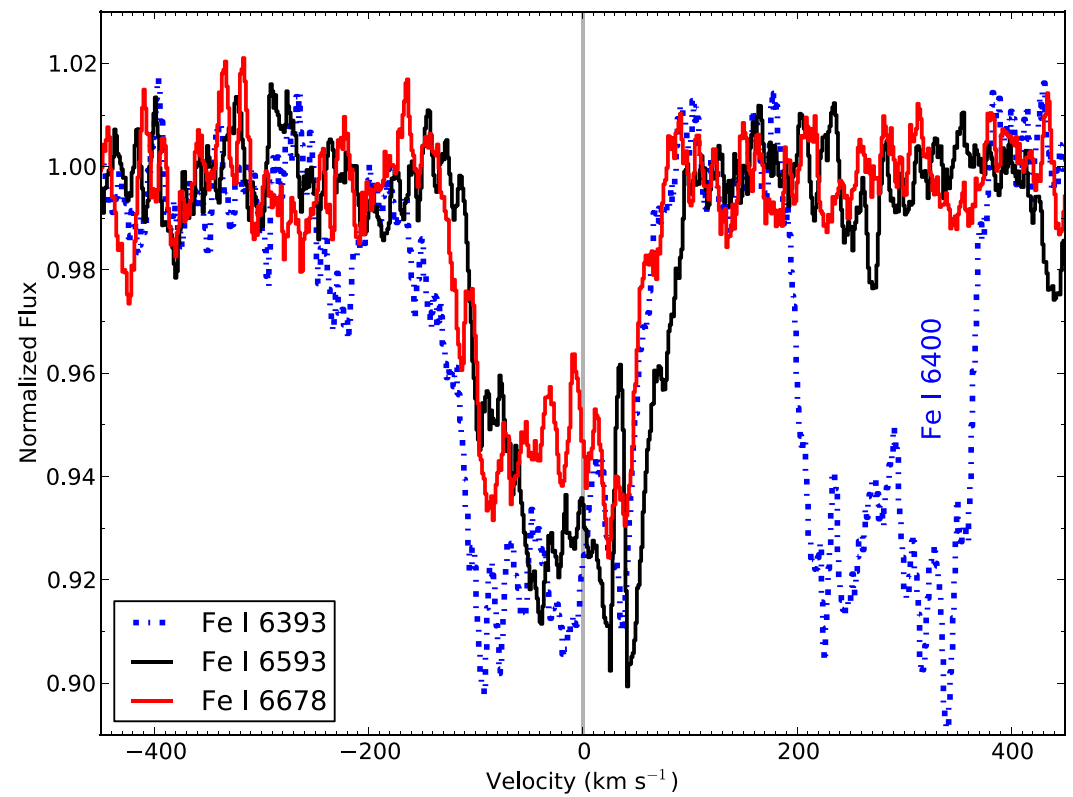

Figure 11. Several Fe I line profiles illustrating the flat-bottomed nature typical of the other metallic lines. The Fe I transitions at $\lambda \lambda 6393,6593$, and 6678 have been shifted to the same velocity, while $\mathrm{Fe}$ I $\lambda 6400$ is offset by $\sim 300 \mathrm{~km} \mathrm{~s}^{-1}$ relative to $\lambda 6393$. These lines all exhibit a profile with a "flat" bottom similar to those observed in FU Orionis (Petrov \& Herbig 2008). For clarity, spectra have been smoothed with a Savitzky-Golay filter, which is similar to a running mean. The vertical gray line is the same as in Figure 9.

(A color version of this figure is available in the online journal.)

forbidden species in emission (such as [N II] and [S II]) typically associated with outflows in YSOs.

The other line worth noting in PTF 10qpf for its kinematic signatures is $\operatorname{Li}$ I $\lambda 6707$. Figure 10 shows the spectral region containing this line, in comparison to V1515 Cyg and V1057 Cyg as well the G2 I standard. The Li I absorption profile has multiple components including a narrow slightly blueshifted component arising from low-velocity gas and a broader absorption extending to $\pm 60 \mathrm{~km} \mathrm{~s}^{-1}$ that is reminiscent of the flat-bottomed profiles seen in other metallic lines in the same spectral region. A similar absorption profile is also seen in Ba II (Figure 9).

Figure 11 illustrates the flat-bottomed nature of many absorption lines in PTF 10qpf. The profiles are reminiscent of those illustrated by Petrov \& Herbig (2008) for FU Ori. When the flat-bottomed lines in PTF 10qpf are cross-correlated against a normal G2 I stellar template, the resulting cross-correlation function is broad $\left(\sim 120 \mathrm{~km} \mathrm{~s}^{-1}\right)$ with several different peaks. In 
some spectral regions, a central peak can be fit by a Gaussian of width $30 \mathrm{~km} \mathrm{~s}^{-1}$, and there are two secondary peaks near the full width of the correlation function, each having Gaussian width $\sim 20 \mathrm{~km} \mathrm{~s}^{-1}$. In other spectral regions, both blue and red, the correlation function has no central peak, but only the double peaks near the limb. To measure the goodness of the cross-correlation, we adopt the Tonry-Davis $R$ value (Tonry $\&$ Davis 1979), which is a ratio of the power in the cross-correlation peak to a measure of the antisymmetry in the correlation. Large $R$ values indicate good cross-correlations. Using a G2 supergiant as a template, the Tonry-Davis $R$ value for PTF 10qpf is $4-6$ (compared to $R>5$ to 15 for V1057 Cyg, which shows broadening and flat-bottomed structure similar to PTF 10qpf in some lines, and $R>20$ for V1515 Cyg, which does not). The cross-correlation results are similar when using V1515 Cyg as a template, rather than the G2 I standard. Employing V1057 Cyg as a template results in a broad and centrally peaked cross-correlation function having width $120-130 \mathrm{~km} \mathrm{~s}^{-1}$.

\subsubsection{Near-infrared Spectroscopy}

NIR spectra of FU Orionis variables resemble the spectra of $\mathrm{K}$ and $\mathrm{M}$ giants/supergiants, in contrast to their earlier spectral type in the optical (Hartmann \& Kenyon 1996). The most prominent features in the NIR spectra of FU Orionis stars are the strong absorption bands from $\mathrm{CO}$ at $2.3 \mu \mathrm{m}$ and the $\mathrm{H}_{2} \mathrm{O}$ vibration-rotation bands at $1.38 \mu \mathrm{m}$ and $1.87 \mu \mathrm{m}$. These absorption bands are clearly present in the outburst spectrum of PTF 10qpf; see Figure 7, which compares spectra from Rayner et al. (2009) of two supergiants (K5 Ib and M5 Ib-II) and a dwarf (M0 V) with PTF 10qpf. There are many prominent absorption features seen in the M0 V spectrum, which are muted or absent in PTF 10qpf and the two supergiant spectra. This is indicative of a low surface gravity environment for PTF 10qpf.

We have attempted to derive a crude NIR spectral type for PTF 10qpf. In addition to its anomalously strong $\mathrm{CO}$ features, PTF 10qpf also possesses prominent $\mathrm{H}_{2} \mathrm{O}$ absorption in the $H$ and $K$ bands; the relative strengths of the $H$-and $K$-band absorptions are unusual, with PTF 10qpf separated from both the dwarf and giant loci for those features (see, e.g., Figure 4 of Covey et al. 2010). We therefore attempted to characterize the NIR spectral type of PTF 10qpf based on the strengths of several $H$-band absorption features: $\mathrm{Mg}_{\mathrm{I}}(1.51 \mu \mathrm{m}), \mathrm{K}_{\mathrm{I}}(1.518 \mu \mathrm{m})$, Al I $(1.67 \mu \mathrm{m})$, and a blend at $1.70 \mu \mathrm{m}$. We compared the strengths of these features to those measured for a suite of dwarf and giant templates by Covey et al. (2010). While this method is sufficient to derive only a crude NIR spectral type for PTF 10qpf, we find that the source's outburst spectrum most closely resembles that of K7 III-M2 III giants (see Figure 7 for some comparisons). It is important to note that the classification scheme from Covey et al. (2010) does not include supergiants; thus, we conclude that PTF 10qpf has a low surface gravity, but we cannot discriminate among luminosity classes I-III.

While the strength of molecular water absorption is greater than that normally observed in giants, this finding is consistent with observations of other FU Orionis stars. Sato et al. (1992) argue that the unusually strong $\mathrm{H}_{2} \mathrm{O}$ absorption seen in $\mathrm{FU}$ Orionis stars can be explained if water that has condensed onto dust grains in the midplane of the disk becomes vaporized during the heating of the disk associated with an FU Orionis eruption. Once this evaporated water is flung vertically out of the disk midplane, it will imprint a strong absorption signature in the NIR spectrum.
Connelley \& Greene (2010) show that the equivalent width (EW) of CO absorption at $2.3 \mu \mathrm{m}$ can be used to identify FU Orionis-like stars when compared to the EW of Na (2.206 and $2.209 \mu \mathrm{m})$ and $\mathrm{Ca}(2.263$ and $2.266 \mu \mathrm{m})$. To compare PTF 10qpf to the stars studied by Connelley \& Greene (2010), we measure the $\mathrm{EW}$ of $\mathrm{Na}, \mathrm{Ca}$, and $\mathrm{CO}$ following the prescription of Chalabaev \& Maillard (1983) with the correction provided by Vollmann \& Eversberg (2006). We measure Na and Ca EWs of $1.56 \pm 0.05$ and $1.07 \pm 0.06 \AA$, respectively, while the EW of CO is $35.6 \pm 0.3 \AA$. These uncertainties reflect the statistical errors in our measurements and likely underestimate the systematic effects associated with these measurements (e.g., the precise placement of the continuum). Nevertheless, the systematic uncertainties are not large enough to alter the general conclusions drawn below. The $\mathrm{CO}$ absorption is well in excess of what would be expected for both late-type dwarfs and giants based on the strength of the $\mathrm{Na}$ and $\mathrm{Ca}$ absorption; however, this excess CO absorption is consistent with other FU Orionis-like stars in the sample of Connelley \& Greene (see their Figure 4).

Prominent $\mathrm{H}$ and $\mathrm{He} \mathrm{I} \lambda 10830$ absorption lines are also visible in the NIR spectra (see Figure 7). However, $\mathrm{H}$ and $\mathrm{He}$ absorption cannot originate in a simple, single-temperature, cool (late $\mathrm{K}-\mathrm{M}$ ) atmosphere. Thus, these lines, though unresolved in our spectra, likely trace an outflow, similar to the Balmer series in the optical. He I $\lambda 10830$ may be a hot-wind indicator, but highresolution spectra are needed to confirm this (Edwards et al. 2006; Kwan et al. 2007).

\section{DISCUSSION AND CONCLUSIONS}

We have shown that the 2010 eruption of $\mathrm{LkH} \alpha$ 188-G4 places it within the small family of FU Orionis-like stars having observed outbursts. LkH $\alpha$ 188-G4/PTF 10qpf exhibits the defining observable characteristics of the FU Orionis class as described by Hartmann \& Kenyon (1996):

1. it exhibited $\mathrm{a} \gtrsim 4$ mag increase in its optical light curve;

2. it is associated with a star-forming region and shows a bright reflection nebula following its eruption;

3. optical spectra of the outburst are consistent with a G-type giant/supergiant;

4. during outburst, the Balmer lines exhibit strong P Cygni profiles, with only $\mathrm{H} \alpha$ showing an emission component, and absorption extending to about $-200 \mathrm{~km} \mathrm{~s}^{-1}$; and

5. the NIR spectra during the eruption resemble those of Mtype giants/supergiants, with strong absorption seen in the $\mathrm{H}_{2} \mathrm{O}$ and $\mathrm{CO}$ bands.

In sharp contrast to the class of YSO outbursts referred to as EX Lupi-like or V1647 Ori-like, PTF 10qpf does not exhibit strong optical/NIR emission lines during outburst.

In the IR, unlike typical M- and K-type supergiants, PTF 10qpf shows prominent absorption lines of $\mathrm{H}$ I $(\mathrm{Br} \gamma, \mathrm{Pa} \beta, \mathrm{Pa} \gamma$, $\mathrm{Pa} \delta$ ) and $\mathrm{He}_{\mathrm{I}} 1.083 \mu \mathrm{m}$. In the optical the $\mathrm{HI}_{\mathrm{I}}$ lines trace an outflow, with clear P Cygni absorption profiles observed in our HIRES spectrum. Our spectral resolution is insufficient to determine whether a similar P Cygni profile is observed in the NIR; however, $\mathrm{H}$ and $\mathrm{He}$ absorption cannot originate in an $\mathrm{M}$ giant/supergiant photosphere. Thus, the $\mathrm{H}$ and $\mathrm{He}$ lines are likely the result of inner-disk region accretion/wind physics.

$\mathrm{LkH} \alpha 188-\mathrm{G} 4$ stands out among the currently known group of FU Orionis-like stars in that there are pre-outburst observations with detections from the optical to the MIR, making $\mathrm{LkH} \alpha 188$ G4 the first FU Orionis-like star with a well-sampled SED prior to eruption. This SED shows that $\mathrm{LkH} \alpha 188-\mathrm{G} 4$ was a Class II 
YSO, which is commonly associated with CTTSs (Lada 1987). A pre-outburst optical spectrum reveals a late-type star, K7-M0, with prominent $\mathrm{H} \alpha$ and $\mathrm{H} \beta$ seen in emission, again consistent with a CTTS. Multiple epochs of optical imaging prior to the outburst reveal that $\mathrm{LkH} \alpha$ 188-G4 exhibited stochastic variability at a level consistent with a CTTS.

One of the most important elements in the study of PTF $10 \mathrm{qpf} / \mathrm{LkH} \alpha 188-\mathrm{G} 4$ is that it is associated with a known, optically revealed, and previously studied YSO. The clear identification of $\mathrm{LkH} \alpha 188-\mathrm{G} 4$ as a CTTS prior to eruption solidifies the interpretation of FU Ori events as enhanced accretion and outflow likely associated with disk-accretion instabilities (Hartmann \& Kenyon 1996). This outburst's association with a previously classified CTTS source shows that FU Ori eruptions are not strictly limited to the most highly embedded Class $0 / \mathrm{I}$ phases of pre-main-sequence evolution. Indeed, the strong winds associated with these events may play an important role in disrupting a YSO's circumstellar envelope. Such a disruption is thought to be a key component of the Class I/II transition.

Continued photometric and spectroscopic monitoring of PTF $10 \mathrm{qpf} / \mathrm{LkH} \alpha$ 188-G4 will fully elucidate its nature. As more and more synoptic surveys come online, the rich legacy of IR observations made by several ground- and space-based observatories (e.g., 2MASS, IRAS, MSX, Spitzer, AKARI, and WISE) over the past two decades will enable the discovery of additional FU Orionis outbursts with well characterized pre-outburst SEDs. These discoveries will in turn allow us to determine if the case of $\mathrm{LkH} \alpha 188-\mathrm{G} 4$ is an outlier or the norm. A better understanding of whether FU Orionis-like eruptions typically occur during the Class I or Class II phase of pre-mainsequence evolution will allow us to place stronger constraints on the triggering mechanism and occurrence rates for these events.

We thank Luisa Rebull for providing the Spitzer/MIPS data for $\mathrm{LkH} \alpha 188-\mathrm{G} 4$ in advance of her publication. We are in debt to John Johnson, John Sebastian Pineda, and Michael Bottom who obtained the HIRES spectrum for us. We thank Cullen Blake, Dan Starr, and Emilo Falco for their efforts to build and maintain PAIRITEL. We thank Meredith Hughes for a fruitful discussion concerning YSO accretion disks and Ryan Foley for information on the spectrophotometric uncertainty associated with Kast spectra. An anonymous referee provided comments that have improved this manuscript.

A.A.M. is supported by the National Science Foundation (NSF) Graduate Research Fellowship Program. K.R.C. acknowledges support for this work from the Hubble Fellowship Program, provided by the National Aeronautics and Space Administration (NASA) through Hubble Fellowship grant HSTHF-51253.01-A awarded by the STScI, which is operated by the AURA, Inc., for NASA, under contract NAS 5-26555. J.S.B. acknowledges support of an NSF-CDI grant-0941742. A.A.M. and C.R.K. were partially supported by NSF-AAG grant-1009991. A.V.F.'s group is grateful for the support of NSF grant AST-0908886, the TABASGO Foundation, Gary and Cynthia Bengier, and the Richard and Rhoda Goldman Fund. The National Energy Research Scientific Computing Center, which is supported by the Office of Science of the US Department of Energy under contract No. DE-AC02-05CH11231, provided staff, computational resources, and data storage for this project.

Some of the data presented herein were obtained at the W. M. Keck Observatory, which is operated as a scientific partnership among the California Institute of Technology, the University of California, and NASA. The Observatory was made possible by the generous financial support of the W. M. Keck Foundation. The authors wish to recognize and acknowledge the very significant cultural role and reverence that the summit of Mauna Kea has always had within the indigenous Hawaiian community. We are most fortunate to have the opportunity to conduct observations from this mountain.

PAIRITEL is operated by the Smithsonian Astrophysical Observatory (SAO) and was made possible by a grant from the Harvard University Milton Fund, a camera loan from the University of Virginia, and continued support of the SAO and UC Berkeley. The PAIRITEL project and those working on PAIRITEL data are further supported by NASA/Swift Guest Investigator Programs NNX09AQ66Q and NNX10A128G. We are grateful for the assistance of the staffs at all of the observatories used to obtain the data.

This research has made use of NASA's Astrophysics Data System Bibliographic Services, the SIMBAD database operated at CDS, Strasbourg, France, the NASA/IPAC Extragalactic Database operated by the Jet Propulsion Laboratory, California Institute of Technology, under contract with NASA, and the VizieR database of astronomical catalogs (Ochsenbein et al. 2000). This publication makes use of data products from the Two Micron All Sky Survey, which is a joint project of the University of Massachusetts and the Infrared Processing and Analysis Center/California Institute of Technology, funded by NASA and the NSF.

Facilities: PO:1.2m (PTF), PO:1.5m, Hale (TripleSpec), Keck:I (HIRES), Shane (Kast), FLWO:2MASS (PAIRITEL)

\section{REFERENCES}

Adelman-McCarthy, J. K., et al. 2008, ApJS, 175, 297

Aspin, C., \& Reipurth, B. 2009, AJ, 138, 1137

Aspin, C., Reipurth, B., Herczeg, G. J., \& Capak, P. 2010, ApJ, 719, L50

Audard, M., et al. 2010, A\&A, 511, 63

Bertin, E., \& Arnouts, S. 1996, A\&AS, 117, 393

Blake, C. H., Bloom, J. S., Latham, D. W., Szentgyorgyi, A. H., Skrutskie, M. F., Falco, E. E., \& Starr, D. S. 2008, PASP, 120, 860

Bloom, J. S., Starr, D. L., Blake, C. H., Skrutskie, M. F., \& Falco, E. E. 2006, in ASP Conf. Ser. 351, Astronomical Data Analysis Software and Systems XV, ed. C. Gabriel et al. (San Francisco, CA: ASP), 751

Bloom, J. S., et al. 2009, ApJ, 691, 723

Bonnell, I., \& Bastien, P. 1992, ApJ, 401, L31

Briceño, C., et al. 2004, ApJ, 606, L123

Cenko, S. B., et al. 2006, PASP, 118, 1396

Chalabaev, A., \& Maillard, J. P. 1983, A\&A, 127, 279

Cohen, M., \& Kuhi, L. V. 1979, ApJS, 41, 743

Connelley, M. S., \& Greene, T. P. 2010, AJ, 140, 1214

Connelley, M. S., Reipurth, B., \& Tokunaga, A. T. 2007, AJ, 133, 1528

Covey, K. R., Lada, C. J., Román-Zúñiga, C., Muench, A. A., Forbrich, J., \& Ascenso, J. 2010, ApJ, 722, 971

Covey, K. R., et al. 2011, AJ, 141, 40

Croswell, K., Hartmann, L., \& Avrett, E. H. 1987, ApJ, 312, 227

Dobashi, K., Bernard, J.-P., Yonekura, Y., \& Fukui, Y. 1994, ApJS, 95, 419

Edwards, S., Fischer, W., Hillenbrand, L., \& Kwan, J. 2006, ApJ, 646, 319

Fedele, D., van den Ancker, M. E., Petr-Gotzens, M. G., \& Rafanelli, P. 2007, A\&A, 427, 207

González-Solares, E. A., et al. 2008, MNRAS, 388, 89

Grankin, K. N., Melnikov, S. Y., Bouvier, J., Herbst, W., \& Shevchenko, V. S. 2007, A\&A, 461, 183

Guieu, S., et al. 2009, ApJ, 697, 787

Hartmann, L., \& Kenyon, S. J. 1996, ARA\&A, 34, 207

Herbig, G. H. 1977, ApJ, 217, 693

Herbig, G. H. 1989, in Proc. European Southern Observatory Conference and Workshop, Vol. 33, ed. B. Reipurth (Garching: ESO), 233

Herbig, G. 2007, AJ, 133, 2679

Herbig, G. H., \& Bell, K. R. 1988, Third Catalog of Emission-Line Stars of the Orion Population (Santa Cruz, CA: Lick Obs.)

Herbig, G. H., Petrov, P. P., \& Duemmler, R. 2003, ApJ, 595, 384

Herter, T. L., et al. 2008, Proc. SPIE, 7014, 70140X 
Horne, K. 1986, PASP, 98, 609

Howard, A. W., et al. 2010, ApJ, 721, 1467

Ishihara, D., et al. 2010, A\&A, 514, A1

Johnson, J. A., et al. 2010, PASP, 122, 149

Kurucz, R. L. 1979, ApJS, 40, 1

Kurucz, R. L. 1993, VizieR Online Data Catalog, 6039

Kwan, J., Edwards, S., \& Fischer, W. 2007, ApJ, 657, 897

Lada, C. J. 1987, in IAU Symp. 115, Star Forming Regions, ed. M. Peimbert \& J. Jugaku (Cambridge: Cambridge Univ. Press), 1

Lada, C. J., \& Wilking, B. A. 1984, ApJ, 287, 610

Law, N. M., et al. 2009, PASP, 121, 1395

Leoni, R., Larionov, V. M., Centrone, M., Giannini, T., \& Lorenzetti, D. 2010, ATel, 2854

Lorenzetti, D., Giannini, T., Larionov, V. M., Kopatskaya, E., Arkharov, A. A., De Luca, M., \& Di Paola, A. 2007, ApJ, 665, 1182

Matheson, T., Filippenko, A. V., Ho, L. C., Barth, A. J., \& Leonard, D. C. 2000, AJ, 120, 1499

Miller, J. S., \& Stone, R. P. S. 1993, Lick Observatory Technical Report No. 66 (Santa Cruz, CA: Univ. California)

Mill, J. D., et al. 1994, J. Spacecr. Rockets, 31, 900

Monet, D. G., et al. 2003, AJ, 125, 984

Munari, U., Milani, A., Valisa, P., \& Semkov, E. 2010, ATel, 2808

Murakami, H., et al. 2007, PASJ, 59, 369

Ochsenbein, F., Bauer, P., \& Marcout, J. 2000, A\&AS, 143, 23

Perley, D. A., et al. 2010, MNRAS, 406, 2473
Petrov, P. P., \& Herbig, G. H. 2008, AJ, 136, 676

Pickles, A. J. 1998, PASP, 110, 863

Rau, A., et al. 2009, PASP, 121, 1334

Rayner, J. T., Cushing, M. C., \& Vacca, W. D. 2009, ApJS, 185, 289

Rebull, L. M., et al. 2011, ApJS, in press (arXiv:1102.0573)

Reipurth, B., \& Aspin, B. 2010, in Evolution of Cosmic Objects Through Their Physical Activity, ed. H. Harutyunyan, A. Mickaelian, \& Y. Terzian (Yerevan: Gitutyun), 19

Rettig, T. W., Brittain, S. D., Gibb, E. L., Simon, T., \& Kulesa, C. 2005, ApJ, 636, 245

Sargent, W. L. W., \& Filippenko, A. V. 1991, AJ, 102, 107

Sato, S., Okita, K., Yamashita, T., Mizutani, K., Shiba, H., Kobayashi, Y., \& Takami, H. 1992, ApJ, 398, 273

Savitzky, A., \& Golay, M. J. E. 1964, Anal. Chem., 36, 1627

Semkov, E., \& Peneva, S. 2010a, ATel, 2801

Semkov, E., \& Peneva, S. 2010b, ATel, 2819

Semkov, E., Peneva, S. P., Munari, U., Milani, A., \& Valisa, P. 2010, A\&A, 523, L3

Skrutskie, M. F., et al. 2006, AJ, 131, 1163

Szeifert, T., Hubrig, S., Schöller, M., Schütz, O., Stelzer, B., \& Mikulášek, Z. 2010, A\&A, 509, 7

Tonry, J., \& Davis, M. 1979, AJ, 84, 1511

Vacca, W. D., Cushing, M. C., \& Rayner, J. T. 2003, PASP, 115, 389

Vogt, S. S., et al. 1994, Proc. SPIE, 2198, 362

Vollmann, K., \& Eversberg, T. 2006, Astron. Nachr., 327, 862 